\title{
Structural Health Monitoring of Precast Concrete Box Girders Using Selected Vibration-Based Damage Detection Methods
}

\author{
Zhengjie Zhou, ${ }^{1}$ Leon D. Wegner, ${ }^{2}$ and Bruce F. Sparling ${ }^{2}$ \\ ${ }^{1}$ Bridge and Structural Design Unit, Road Service Division, King County Department of Transportation, KSC-TR-0242, \\ 201 South Jackson Street, Seattle, WA 98104-3856, USA \\ ${ }^{2}$ Department of Civil and Geological Engineering, University of Saskatchewan, 57 Campus Drive, Saskatoon, SK, Canada S7N $5 A 9$
}

Correspondence should be addressed to Leon D. Wegner, leon.wegner@usask.ca

Received 15 October 2009; Revised 30 January 2010; Accepted 23 March 2010

Academic Editor: Yi Qing Ni

Copyright ( $\odot 2010$ Zhengjie Zhou et al. This is an open access article distributed under the Creative Commons Attribution License, which permits unrestricted use, distribution, and reproduction in any medium, provided the original work is properly cited.

\begin{abstract}
Precast, prestressed concrete box girders are commonly used as superstructure components for short and medium span bridges. Their configuration and typical side-by-side placement make large portions of these elements inaccessible for visual inspection or the application of nondestructive testing techniques. This paper demonstrates that vibration-based damage detection (VBDD) is an effective alternative for monitoring their structural health. A box girder removed from a dismantled bridge was used to evaluate the ability of five different VBDD algorithms to detect and localize low levels of spalling damage, with a focus on using a small number of sensors and only the fundamental mode of vibration. All methods were capable of detecting and localizing damage to a region within approximately 1.6 times the longitudinal spacing between as few as six uniformly distributed accelerometers. Strain gauges configured to measure curvature were also effective, but tended to be susceptible to large errors in near support damage cases. Finite element analyses demonstrated that increasing the number of sensor locations leads to a proportional increase in localization accuracy, while the use of additional modes provides little advantage and can sometimes lead to a deterioration in the performance of the VBDD techniques.
\end{abstract}

\section{Introduction}

Although there has been a growing awareness of the declining state of the civil infrastructure in North America for several decades, recent catastrophic bridge failures have highlighted both the severity of the problem as it relates to bridges, and the inadequacy of current inspection and monitoring practices to assess their condition [1]. More objective means for monitoring the structural health of bridges have been pursued for some time by the research community. While a number of local nondestructive evaluation (NDE) methods $[2,3]$ or global response-based methods [4-6] are either in current use or are at various stages of development, the application of a specific structural health monitoring (SHM) technique will be most successful when its capabilities are closely matched to the features and requirements of a particular bridge component.

Vibration-based damage detection (VBDD) may be particularly well-suited to assessing the condition of precast, prestressed concrete box girders. This type of girder is commonly employed as a superstructure component for short and medium span bridges. Available in standard cross sectional dimensions and lengths, they are typically used in simple span construction, with multiple units arranged side-by-side and connected by shear keys or in some other manner to facilitate load sharing. Large portions of these elements remain inaccessible to inspection either visually or by common NDE methods. VBDD techniques, on the other hand, are capable of assessing the condition of an entire structural component simultaneously and are not limited to the interrogation of accessible regions. They have also been shown to be capable of identifying the presence and location of low levels of damage on other types of simple-span structures [7]. The capabilities of VBDD methods therefore appear to closely match the monitoring requirements of precast concrete box girders, although the application of VBDD methods to these components has apparently not been reported in the literature. 


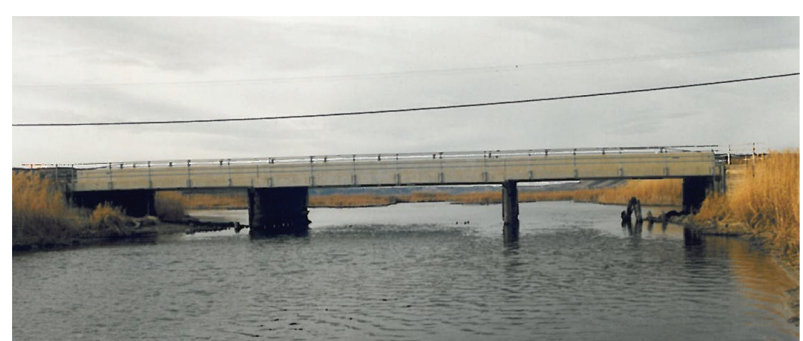

FIGURE 1: Photograph of the bridge prior to girder replacement.

To clarify the principle underlying VBDD techniques, the vibration characteristics of a structure (e.g., its natural frequencies and mode shapes) can be considered to be a global response signature that can be used as the basis for assessing its condition because they contain embedded information about the structure's inherent properties. Changes in the structural condition will be reflected in the vibration signature, making it theoretically possible to identify the presence of damage by tracking changes to that signature. On this basis, numerous VBDD indices have been proposed [6, $8,9]$, including those that rely on shifts in natural frequencies [10-12], direct changes to mode shapes [13,14], changes to mode shape curvatures $[15,16]$, changes to measurementderived flexibility or stiffness matrices $[17,18]$, changes to modal strain energy $[19,20]$, and numerical model updating $[21,22]$.

Having been applied to numerous different types of structures $[17,23,24]$, including real bridge structures [12, $20,25-30]$, VBDD has yet to find widespread acceptance as an SHM tool for bridges. In fact, the authors are not aware of a single case in which these techniques are being routinely applied as a component of a systematic monitoring strategy for bridge management; successful application remains limited to investigations by experts in a research context. However, as a relatively simple component in form as well as application, precast concrete box girders may be particularly amenable to the application of VBDD.

The objective of the current study was to assess the ability of VBDD techniques to identify damage on precast, prestressed concrete box girders. It should be noted, though, that the results can be applied to any simple-span beam-like structural component. In order to consider an approach that could be practically applied in the field, the focus was on detecting low (incipient) levels of damage using a small number of sensors and only the fundamental mode of vibration. The study was carried out in the laboratory using a box girder that had been reclaimed from a dismantled bridge. Given that arrangement of these components in the field is very similar to the laboratory setup, it is believed that the findings of the laboratory investigation can be transferred directly to the field, provided that similar experimental techniques can be applied in the field application and that care is taken to mitigate the effects of environmental influences. Experimental data were supplemented by finite element analyses to explore additional considerations, including a larger number of damage scenarios, the influence of the number of measurement points used to define mode shapes, and the influence of accounting for additional vibration modes.

\section{Experimental Study}

The study was performed using a precast, prestressed concrete box girder that was removed from a bridge on Provincial Highway No. 56 over the Qu'Appelle River in southern Saskatchewan. Figure 1 shows the bridge prior to girder replacement. Originally constructed in 1972, the bridge featured two $12.2 \mathrm{~m}$ end spans and a central $15.2 \mathrm{~m}$ span with nine box girders arranged side-by-side on each span, supported by timber bents and piles. The girders were replaced in 2001 due to extensive corrosion after less than 30 years in use. At that time, one of the dismantled 12.2 $\mathrm{m}$ long girders was transported to the structural laboratory at the University of Saskatchewan for testing (Figure 2(a)). The standard double box girder had a $1216 \times 508 \mathrm{~mm}$ cross section, as seen in Figure 2(b). It was simply supported in the laboratory at each of its four corners, resulting in a longitudinal span of $11.9 \mathrm{~m}$.

The procedures used for the experimental study were intended to simulate methods that could be practically applied in a field application. This included the use of a relatively small number of sensors and of "output-only" modal analysis methods, which do not require the measurement of the excitation force and, therefore, do not make use of the frequency response function (FRF). Field applications would typically involve the measurement of the dynamic properties of the girder on a periodic basis, likely at a similar time of year to avoid large temperature differences between successive tests which could introduce additional variability in the vibration characteristics [31].

The experimental test procedure consisted of measuring the initial dynamic properties of the girder, and then incrementally inducing a succession of new damage states and measuring the dynamic properties associated with each new state. The baseline dynamic signature for a particular damage state was taken to be that measured for the previous state of damage. Well-defined "spalling" damage was induced into the girder by physically removing small square blocks of concrete, $150 \times 150 \mathrm{~mm}$ in plan and $30 \mathrm{~mm}$ deep, from the top surface of the deck, as shown in Figure 3. This was done incrementally at the twelve locations shown in Figure 4 , in which damage states are numbered according to the sequence in which they were introduced. The twelve cases represented a wide variety of longitudinal and transverse locations to test the damage localization capability of the VBDD techniques over a large range of possible damage locations. Each incremental damage state corresponded to a local reduction in flexural rigidity of approximately $2.5 \%$.

Common forms of damage experienced by this type of structural element include localized corrosion, surface spalling, and abrasion of the wearing surface. The type of damage induced experimentally was considered to be representative of any form of damage that causes a localized reduction in the flexural rigidity. Therefore, the findings 


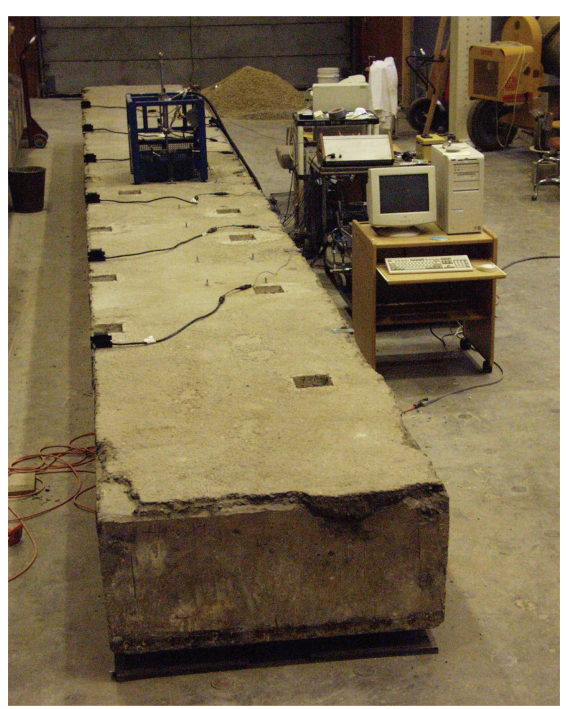

(a)

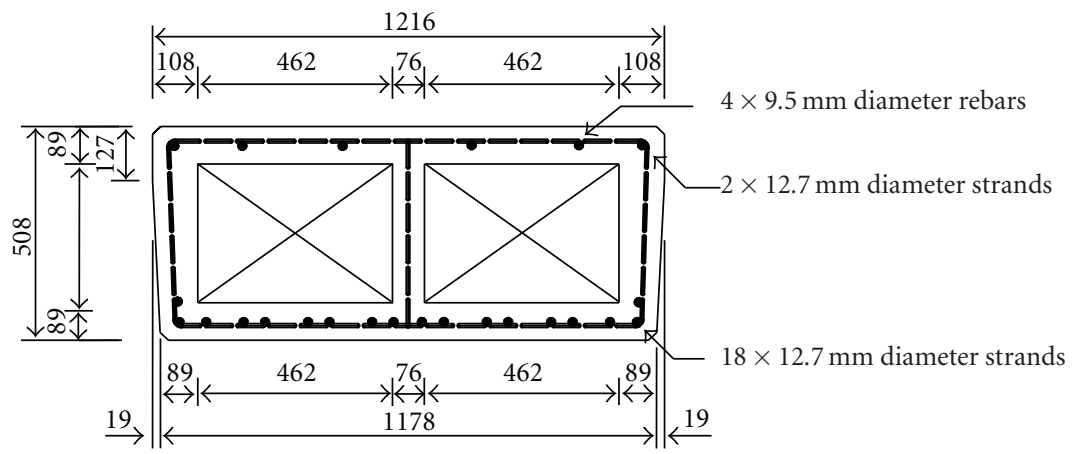

(b)

Figure 2: The prestressed concrete box girder used for the experimental study: (a) photograph of the girder set up for testing in the laboratory, and (b) transverse cross section (dimensions in $\mathrm{mm}$ ).

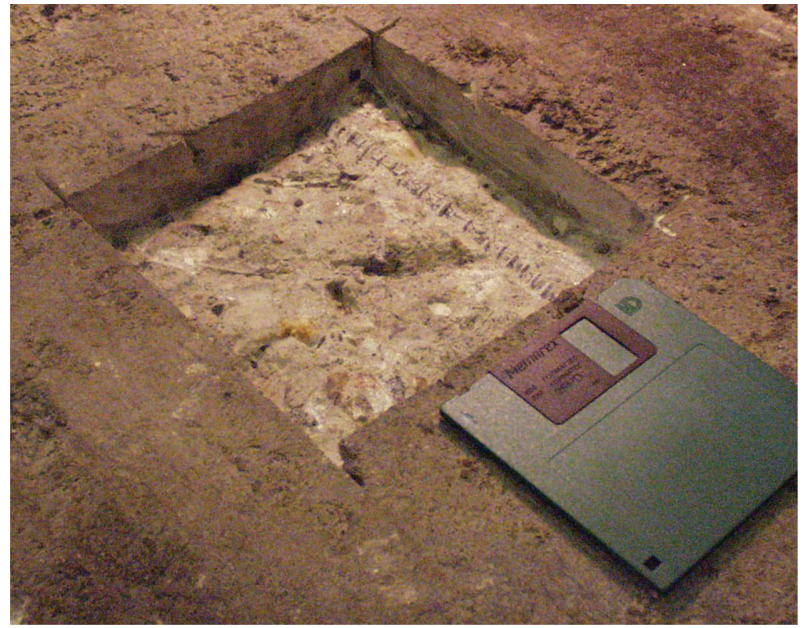

FIGURE 3: Photograph of damage induced on the girder.

should not be considered to be limited to the specific type of damage imposed here.

A hydraulic shaker, seen in Figure 2(a), was anchored directly to the top surface of the girder and used to induce dynamic excitation. The signal for the shaker was generated using LabView software [32] implemented on a personal computer. As mentioned above, the excitation force was not measured.

The dynamic response was measured using two different types of sensors: accelerometers and strain gauges. Data were acquired using a 12-bit data acquisition system (National Instruments SCXI 1001, LabView 6i). The single axis accelerometers (EpiSensor ES-U, Kinemetrics Inc., Pasadena, CA) were configured for a maximum range of $\pm 0.5 \mathrm{~g}$ and a precision of $0.00025 \mathrm{~g}$ and were oriented to measure vertical acceleration. Six accelerometers were bonded to the top surface of the girder, first along one side and then the other, at evenly spaced longitudinal intervals of $1.7 \mathrm{~m}$, as indicated in Figure 4. Vertical displacements at each accelerometer location were obtained by integrating the acceleration signal twice to obtain first velocity and then displacement, incorporating appropriate digital filters at each stage to remove baseline drift. Strictly speaking, the acceleration signals could have been used directly, without conversion to displacements, since the amplitudes of the two signals are exactly proportional at any given frequency. In practice, however, the numerical integration over time and the additional filtering that was employed served to attenuate noise in the signals, thus resulting in more repeatable natural frequencies and mode shapes. Each displacement-time series was then scaled using a Parzen window function [33] to reduce leakage before applying a Fast Fourier Transform (FFT) to obtain a Fourier spectrum of the response amplitude.

The use of strain gauges permitted a comparison of the performance of the VBDD techniques using data from both sets of sensors. Electrical resistance foil strain gauges (model PL-90-11, Tokyo Sokki Kenkjujo Co. Ltd., Japan) with a $90 \mathrm{~mm}$ gauge length and $120 \mathrm{ohm}$ resistance were bonded to the vertical side surfaces of the girder in vertically aligned groups of three, oriented to measure longitudinal strain, at the same six longitudinal locations as used for the accelerometers along each side (see Figure 4). In a field application, the side faces would not be accessible for post-construction application of the gauges, since the girders are placed immediately adjacent to each other. However, the gauges could be installed in well-controlled conditions in the precast plant prior to placement of the girders. The strain profile through a vertical cross section at each location was obtained by fitting a least-squares regression line through the three 


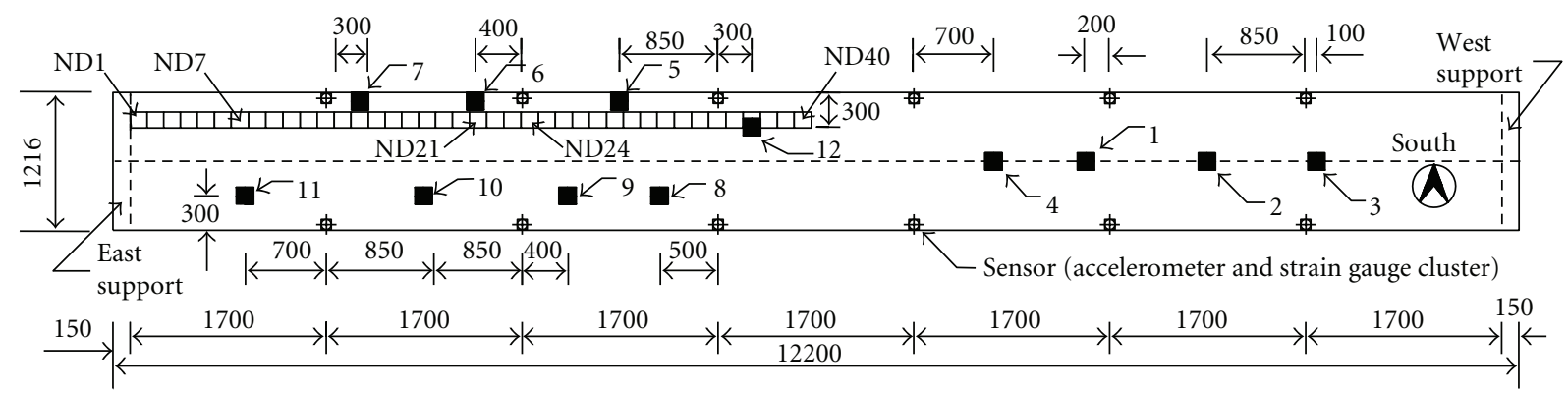

FIGURE 4: Schematic plan of girder showing locations of experimental damage cases (numbered and dimensioned black squares), numerical damage cases (open squares, selected cases labelled ND1, etc.), and sensors (dimensions in $\mathrm{mm}$ ).

TABLE 1: Comparison of FE and experimental natural frequencies and mode shapes for the undamaged system.

\begin{tabular}{lcccc}
\hline Parameter & & Mode & & \\
& 1 (flexural) & 2(flexural) & & \\
Natural Frequencies (Hz) & & & 31.7 & 57.9 \\
$\quad$ Experimental model & 7.61 & 26.3 & 36.46 & 58.95 \\
Finite element model & 7.58 & 27.23 & 15.0 & 1.8 \\
Relative error (\%) & -0.4 & 3.5 & 0.9882 & 0.9865 \\
\hline Modal Assurance Criteria: & 0.9999 & 0.9967 & \\
\hline
\end{tabular}

individual strain measurements at each instant in time. This linear profile was used to calculate the bending curvature at each location. Once again, a Parzen window function was applied to the curvature-time series to reduce leakage, and an FFT was used to obtain spectrum data for the curvatures. The fundamental mode curvatures at measurement points were used to calculate the change of curvature vector, $\Delta \phi^{\prime \prime}$, as described in Section 4, for use with the mode shape curvature method. Intermediate values of $\Delta \phi^{\prime \prime}$ between measurement points were generated using a Bézier interpolation technique [34].

Natural frequencies were identified from the average of ten root-mean-square ( $\mathrm{rms}$ ) normalized frequency response spectra. These were obtained from tests during which data were acquired at a rate of 150 samples per second for a period of 220 seconds while the shaker subjected the girder to a white noise excitation. After natural frequencies had been identified, a resonant harmonic excitation was applied at each of the identified natural frequencies in sequence to accurately measure the corresponding mode shapes. During this procedure, each set of data was acquired at 300 samples per second for 21 seconds. The average of ten mode shape measurements was used in subsequent damage detection algorithms. While only the fundamental mode was used for damage detection experimentally, the first four modes were used for calibration of the finite element (FE) model described below.

\section{Numerical Simulations}

A finite element (FE) model of the girder was prepared and calibrated to match the experimentally measured dynamic properties, with the purpose of investigating the influence of several factors that could not easily be studied experimentally on the performance of the VBDD techniques. These factors included the number of measurement points and modes used, as well as a wider range of damage locations. The commercial FE analysis package ANSYS [35] was used to perform eigenvalue analyses of the girder when subjected to several states of damage in order to generate the natural frequencies and mode shapes of the system at these various states. The girder was divided into 8node 3-dimensional isoparametric brick elements. A total of 84 elements were used longitudinally (including two elements beyond the supports at each end to model the end plates), with the top and bottom flanges divided into twenty-four elements transversely, and two (for the bottom plate) or three (for the top plate) elements through the thickness. The vertical webs were divided into five elements vertically and two elements through the thickness, and the prestressing tendons were modelled using linear truss elements.

Boundary conditions were imposed at the four support nodes, each located $150 \mathrm{~mm}$ from the ends and $50 \mathrm{~mm}$ from the sides, restraining them against movement in the vertical direction. In addition, one of these nodes was restrained in both the longitudinal and transverse directions, a second in the longitudinal direction, and a third in the transverse direction, preventing rigid body movement of the system. Elastic and section properties of the physical components were applied as model parameters.

The model was calibrated to the four lowest natural frequencies and mode shapes of the undamaged physical system by adjusting the value used for Young's modulus 
of the concrete. Table 1 shows that, with the exception of the third (torsional) mode, good agreement between predicted and measured natural frequencies was achieved; in addition, that the modal assurance criterion (MAC) values [36] comparing the first four measured and calculated mode shapes were excellent. A separate study found that the torsional mode was very sensitive to slight differences in support conditions. These differences could not be eliminated because it was not possible to adjust the supports perfectly so that the weight of the girder was equally distributed to the four supports. The good agreement otherwise is an indication that the model could be relied upon to provide a reasonably good estimate of the performance of the VBDD methods.

Once the model had been calibrated, damage to the girder was simulated by eliminating three transversely adjacent elements from the top surface of the girder, resulting in the removal of a region $149 \mathrm{~mm}$ long, $150 \mathrm{~mm}$ wide and $30 \mathrm{~mm}$ thick-a size similar to that investigated experimentally. A total of 40 damage cases were simulated. For these, the transverse location of damage remained centred $0.225 \mathrm{~m}$ from one side of the girder, while the central longitudinal location of the removed elements was varied from 0.074 to $5.876 \mathrm{~m}$ relative to the support at $0.149 \mathrm{~m}$ intervals (see Figure 4).

In order to simulate the acquisition of measured data from a limited number of sensors attached to a physical system, vertical modal displacements were extracted from the FE-generated eigenvectors at a small number of uniformly spaced "measurement" points along the longitudinal edges of the girder. It is noted that the location of simulated measurement points along the girder edges matched what was done experimentally, an arrangement that enabled the identification of torsional modes. Evaluation of the influence of the number of measurement points was facilitated by investigating three cases: one in which seven measurement points were used, one in which 15 measurement points were used, and a well-defined reference case in which 79 measurement points were used. In each case, the measurement points were uniformly distributed along the length of the girder. It should be noted that the smallest number of measurement points did not match the six used experimentally; as described above, the girder was divided into 80 elements along the span length $(11.9 \mathrm{~m})$ to accommodate the desired damage size, and this was compatible with seven uniformly spaced measurement points, but not six.

Regardless of the number of simulated measurement points, the modal displacement vectors $\phi$ used for calculations all contained 81 components, which included components of zero at each support. When seven or 15 simulated measurement points were used, the additional components between measurement points were interpolated as described in the following section. To clarify, when seven simulated measurement points were used, the modal displacements at nine intermediate points between each pair of adjacent measurement points were interpolated, while when 15 simulated measurement points were used, modal displacements at four intermediate points were interpolated.
While the focus of the study was on the performance of the damage detection techniques using only the fundamental mode, the use of the first three flexural modes was also investigated to ascertain the influence of the number of modes considered on the performance of the VBDD methods.

In order to establish the validity of the numerical calculations and justify the extension of the study to a larger number of damage cases using FE simulations, comparison of the numerical and experimental results for a representative damage case is presented in Appendix A.

\section{Damage Detection Methods}

Five of the most common VBDD techniques available in the literature were applied. These included the change in mode shape (CMS) method [37], the mode shape curvature (MSC) method [15], the change in flexibility (CF) method [17], the damage index (DI) method [38], and the change in uniform flexibility curvature (CUFC) method [28]. Among these, the MSC, DI, and CUFC methods-all calculated using mode shape curvatures-produced virtually identical results. For this reason, and for the sake of brevity, the DI method was selected as being representative of these three methods, with results for the other two curvature-based methods not provided. The exception to this is the results of the MSC method obtained using curvatures that were measured directly using a system of strain gauges presented in Section 5.2. It should be noted that all of the methods investigated are based only on measured data and do not require the use of a numerical model. This is considered to be an important feature of methods that could be practically applied to a large inventory of short and medium span bridges, for which the preparation of an FE model is generally unwarranted.

Each of the techniques applied in this study makes use of the measured mode shapes for the structure in baseline and damaged conditions, designated by modal amplitude vectors $\phi$ and $\phi^{*}$, respectively. For the present study, these vectors corresponded to vertical modal amplitudes defined at a relatively small number of uniformly distributed measurement points along the girder, supplemented by additional interpolated modal amplitudes between measurement points. Since the scale of a mode shape is arbitrary by definition, mode shape vectors must be normalized to a common basis, thereby minimizing the difference between damaged and undamaged mode shapes in an averaged sense and accentuating localized changes caused by damage. Vectors were therefore scaled to possess a unit norm (i.e., $\phi^{\mathrm{T}} \boldsymbol{\phi}=1$ ). In effect, this approach may be considered equivalent to mass orthonormalization assuming a uniform distribution of mass. Such an assumption is particularly justifiable when the structure is a prismatic flexural member for which modal amplitudes are defined at uniformly distributed points along the length of the span, as was the case for the current investigation.

Formulations for the VBDD techniques are provided in detailed form in the literature cited, but are briefly summarized here. 
4.1. Change in Mode Shape Method (CMS). The change in mode shape vector, $\Delta \phi$, was calculated by taking the simple difference between damaged and undamaged mode shapes

$$
\Delta \phi=\left|\phi^{*}\right|-|\phi|,
$$

where the absolute value symbols indicate evaluation of the absolute value of each component of the vector.

4.2. Mode Shape Curvature Method (MSC). The MSC method considers the increase in modal curvature caused by damage:

$$
\Delta \phi_{i}^{\prime \prime}=\left|\phi_{i}^{* \prime \prime}\right|-\left|\phi_{i}^{\prime \prime}\right|,
$$

where the double prime notation indicates the second spatial derivative and the subscript $i$ refers to the $i$ th mode. Again, evaluation of absolute values is carried out on each component of the vector. If multiple modes are used, the sum of differences in curvature may be employed as a damage indicator, as expressed by

$$
\Delta \phi^{\prime \prime}=\sum_{i=1}^{n} \Delta \phi_{i}^{\prime \prime},
$$

where $n$ is the number of modes used.

4.3. Change in Flexibility Method (CF). As originally formulated [17], the CF method involves estimating the flexibility matrices for the undamaged and damaged structure, $\mathbf{F}$ and $\mathbf{F}^{*}$, respectively, from one or more of the lower vibration modes as follows

$$
\begin{gathered}
\mathbf{F} \approx \sum_{i=1}^{n} \frac{1}{\omega_{i}^{2}} \phi_{i} \phi_{i}^{T}, \\
\mathbf{F}^{*} \approx \sum_{i=1}^{n} \frac{1}{\omega_{i}^{* 2}} \phi_{i}^{*} \phi_{i}^{* T},
\end{gathered}
$$

in which $\omega_{i}$ is the angular frequency of the $i$ th mode and $n$ is the number of measured modes. The change in flexibility matrix, $\Delta \mathbf{F}$, is then calculated as

$$
\Delta \mathbf{F}=\mathbf{F}^{*}-\mathbf{F} .
$$

The parameter $\bar{\delta}_{j}$, the maximum of the absolute values of the elements in column $j$ of $\Delta \mathbf{F}$, is taken as a measure of the change of flexibility at point $j$ of the structure. The maximum change of flexibility then provides an indication of the location of damage.

4.4. Modified Change in Flexibility Method (MCF). For the present study, a slight modification to the CF method is proposed on the basis of physical arguments. Since column $j$ of the flexibility matrix represents the deflected shape of the structure when a unit load is applied at location $j$, the change in flexibility at a location of damage should always be positive since the deflection under a unit load at that location should always increase when damage is inflicted at that point.
Therefore, it is proposed that the maximum positive value of elements in column $j$ be used as the damage indicator, rather than the maximum of the absolute values. The modified approach was observed to improve the performance of the method, particularly when experimental data were used. Appendix B compares the distributions produced by the change in flexibility method in original and modified forms for a representative damage case.

4.5. Damage Index Method (DI). The DI method is based on local changes in modal strain energy. In discrete form, the index takes the following form

$$
\beta_{j i}=\frac{\left(\varphi_{j i}^{\prime \prime *}\right)^{2}+\sum_{k=1}^{m}\left(\varphi_{k i}^{\prime \prime *}\right)^{2}}{\left(\varphi_{j i}^{\prime \prime}\right)^{2}+\sum_{k=1}^{m}\left(\varphi_{k i}^{\prime \prime}\right)^{2}} \times \frac{\sum_{k=1}^{m}\left(\varphi_{k i}^{\prime \prime}\right)^{2}}{\sum_{k=1}^{m}\left(\varphi_{k i}^{\prime \prime *}\right)^{2}}=\frac{\mathrm{NUM}_{j i}}{\operatorname{DEN}_{j i}}
$$

in which $\varphi_{j i}^{\prime \prime}$ corresponds to the $j$ th element of the modal curvature vector $\phi^{\prime \prime}$ for the $i$ th mode. If more than one mode is used, a single index for each location, $j$, is formed by

$$
\beta_{j}=\frac{\sum_{i=1}^{n} \operatorname{NUM}_{j i}}{\sum_{i=1}^{n} \operatorname{DEN}_{j i}} .
$$

The index is normalized by considering it to be a normally distributed random variable

$$
Z_{j}=\frac{\beta_{j}-\mu_{\beta}}{\sigma_{\beta}},
$$

where $\mu_{\beta}$ and $\sigma_{\beta}$ are the mean and standard deviation of damage indices, respectively. Damage indices falling two or more standard deviations from the mean (i.e., $Z_{j} \geq 2$ ) are defined as being indicative of a possible damage location.

To further clarify the implementation of the methods used, it should be observed that the curvature-based methods (DI, MSC, CUFC) require estimates of modal curvature at measurement points. Elements of the modal amplitude vectors initially corresponded to values defined at a relatively small number of measurement points. In order to generate a sufficiently well-defined displacement profile required to facilitate a more accurate estimation of curvature, the displacement profile at up to nine points between each pair of adjacent sensor locations was estimated using a natural cubic spline interpolation scheme, which enforced continuity of the second derivative at measurement points and a zero second derivative at the simple supports. A similar approach has been reported in the literature [20, 39, 40]. Modal curvatures were then explicitly obtained from the resulting spline equations for use with the MSC and DI methods. Although interpolation was strictly not required to apply the MCF and CMS methods, its use was not found to impair the performance of the methods, and could actually improve their localization capabilities by permitting predicted damage locations to lie between measurement points. Therefore, the same interpolated mode shapes were used for all VBDD methods. 


\section{Results and Discussion}

\subsection{Performance Using Only a Small Number of Measurement Points}

5.1.1. Typical VBDD Parameter Distributions. The distributions of the VBDD parameters calculated for experimental damage case 4 are provided in Figure 5, in which the locations of the accelerometers are indicated by the vertical gridlines. To facilitate the plotting of all distributions on a common scale, all parameters have been normalized in a manner similar to that used by the DI method; that is,

$$
\hat{P}_{i}=\frac{P_{i}-\mu_{P}}{\sigma_{P}},
$$

where $\hat{P}_{i}$ represents the normalized parameter at location $i, P_{i}$ is the unnormalized VBDD parameter (i.e., CMS and $\mathrm{MCF})$ at that location, $\mu_{P}$ is the mean of parameter values at all locations along the girder length, and $\sigma_{P}$ is the standard deviation of parameter values.

The distributions shown in Figure 5 are typical of those produced for damage cases that were located approximately within the middle two-thirds of the span. In these cases, a clear maximum peak was produced at or near the same location by all methods, which corresponded to one of the two measurement points adjacent to the damage location, although not necessarily the nearest one. In addition, it is noted that the DI method produced a piecewise linear distribution between interior measurement points, which is a consequence of the cubic spline interpolation procedure used to estimate curvature values between measurement points. As such, the curvature-based methods are only capable of producing peaks at measurement points.

5.1.2. Effect of Proximity of Damage to a Support. The distributions associated with a sequence of damage locations moving from left to right (east to west) along the girder are illustrated in Figure 6. It is apparent that the distributions associated with near support damage cases (cases 11, 10, and 3) featured characteristics that clearly distinguished them from the other damage cases. These characteristics differed according to the method applied. For the CMS method, distributions for near support damage cases featured multiple undulations, the highest peak of which was located near the damage. For the MCF method, two significant peaks of similar magnitude were produced. The higher of the two peaks was not always the one closest to the damage (e.g., case 3 ), but one of the peaks was located near the damage while no peaks were located in the vicinity of the opposite support. For the DI method, near support damage cases featured multiple peaks, the most prominent of which was not located near the damage. As a result, the DI method was unable to locate near support damage cases, and the predicted damage location was erroneous when damage was located near a support.

As damage moved slightly farther from the supportgenerally beyond the first measurement point-a clear,

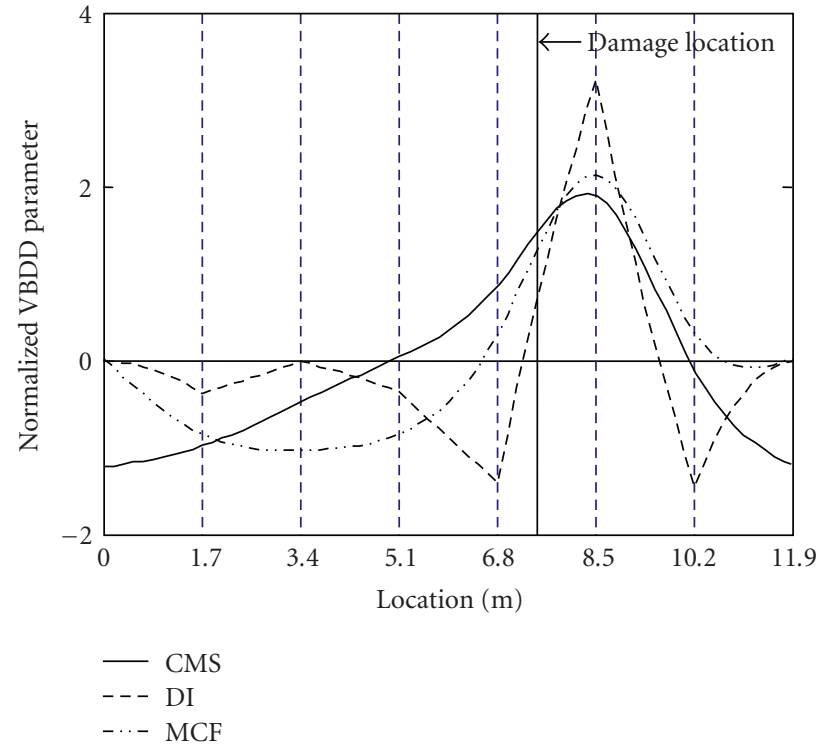

FIGURE 5: Distributions of normalized VBDD parameters for experimental damage case 4.

dominant peak was produced by all VBDD indices, as seen, for example, with damage case 2, located $2.55 \mathrm{~m}$ from the west (right) support. This feature characterized the distributions produced by all non-near support damage cases. On the other hand, damage case 10 was located the same distance $(2.55 \mathrm{~m})$ from the east (left) support, and still featured the near support characteristics just described. Damage case 6 , however, located only slightly farther $(3.0 \mathrm{~m})$ from the east (left) support, produced a single dominant peak by all methods. This indicates that slight differences in support conditions affected the near support length within which distributions featured multiple peaks. For this particular study, it was not possible to distribute the girder's weight evenly to the simple supports at the four corners, making support conditions slightly different at each of the four support locations.

The above discussion indicates that damage located near supports is more challenging to identify by virtue of the appearance of multiple peaks in the VBDD distributions. However, the following results were consistently observed: when multiple peaks were produced by the CMS and MCF methods, and when the three VBDD parameters differed in the location of the maximum peak, the damage was located close to a support. In these cases, the MCF method consistently indicated which of the supports damage lay close to, and the CMS method always produced a maximum peak near the damage location, even when other peaks were present. The CMS method was, therefore, the most reliable among the methods investigated when localizing near support damage cases. The simultaneous application of the other methods, however, can provide an added level of confidence in the predicted damage location, particularly in terms of distinguishing near support damage cases from others. 


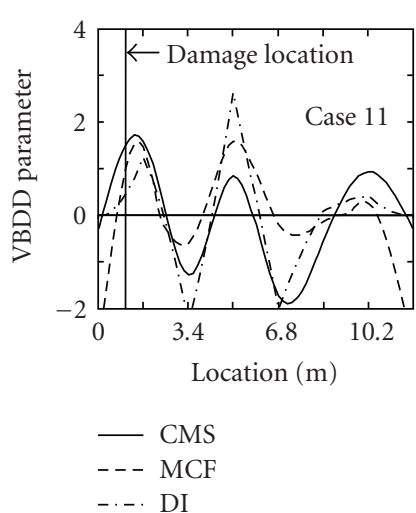

(a)

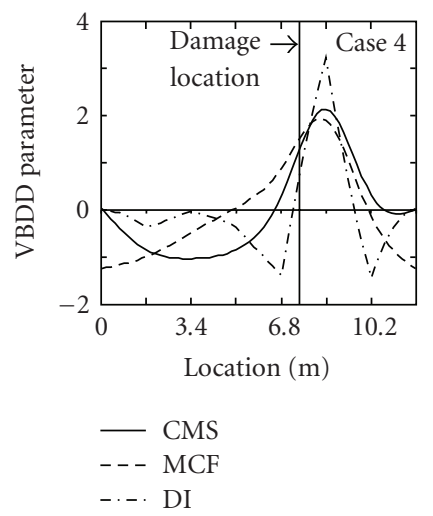

(e)

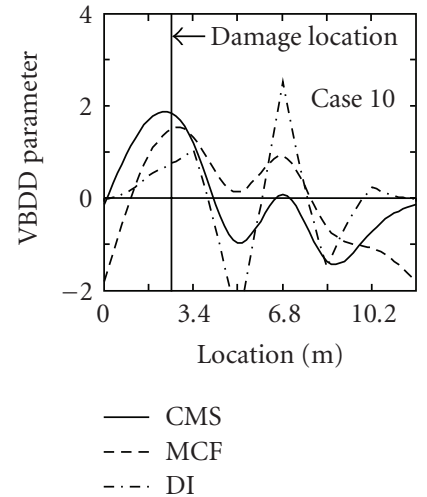

(b)

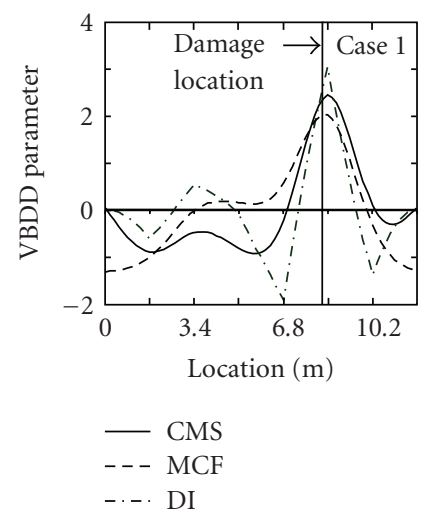

(f)

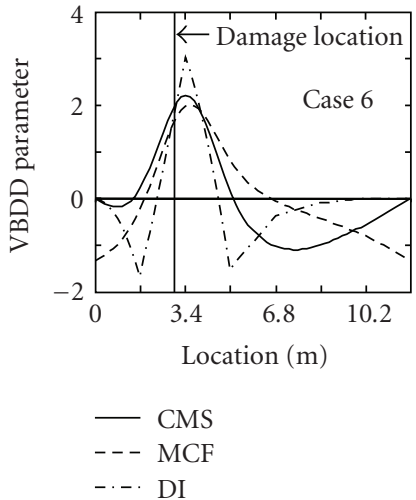

(c)

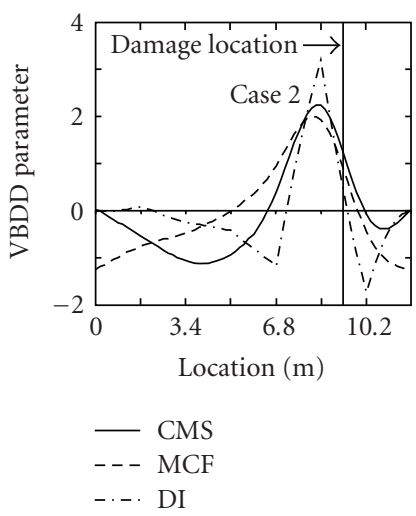

(g)

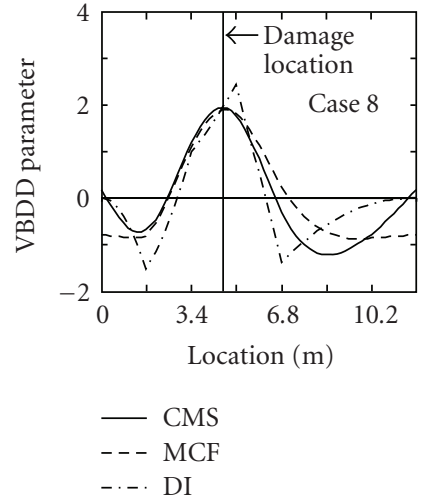

(d)

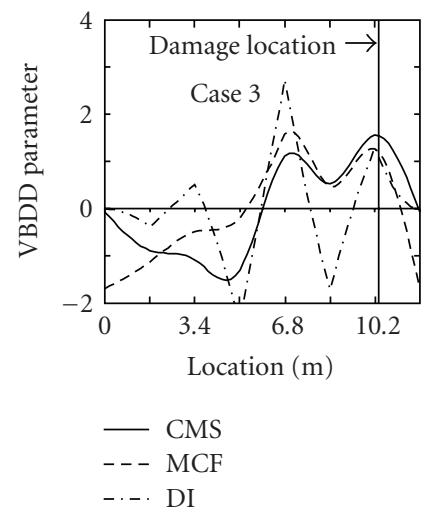

(h)

FIgURE 6: Normalized VBDD distributions for a sequence of experimental damage cases, with damage moving from east to west (left to right) along the girder.

5.1.3. Localization Accuracy. The performance of each of the methods in terms of its ability to identify the damage location is summarized in Figure 7, which plots the predicted versus actual locations of damage for all 12 experimental damage cases and all 40 numerical damage cases. In these figures, labelled gridlines correspond to the experimental measurement point locations, whereas the additional dotted gridlines correspond to the numerical measurement points. In addition, different symbols have been used to distinguish predictions that corresponded to a single clear peak in the distribution from those in which a second peak with at least $75 \%$ of the magnitude of the first also occurred. When the second largest peak appeared nearer to the damage location, that peak is plotted as the predicted location. In some cases, the peak that occurred nearer to the damage location did not meet the $75 \%$ threshold. In these cases, the height of the second peak relative to the first is provided as a percentage value next to the corresponding data point.

Comparing the performance of the three methods shown in Figure 7, it is apparent that the CMS method was most consistent in locating the damage without ambiguity. The numerical localization predictions for this method (Figure 7(a)) were also more accurate than the experimental predictions. This latter result is expected, since numerical data were free from the uncertainties inherent in all experimental data. The MCF method (Figure 7(b)) tended to produce ambiguous results for near support damage cases. These ambiguities resulted from a second peak of roughly the same magnitude appearing farther from the support. Figure 7(c) clearly shows that the DI method was only capable of predicting damage to be located at measurement points, both experimentally and numerically.

Figures 7(a) and 7(b) also show that the use of interpolation to estimate modal displacements between measurement points allowed the CMS and MCF methods to predict damage locations between measurement points. In the case of the CMS method, interpolation permitted the localization of damage with greater accuracy over a wider range of damage locations when numerically simulated data were used. The use of interpolation is therefore advisable even in cases where it is not specifically required to estimate curvature. It also suggests that further benefit could be derived from the identification of interpolation techniques that are best able to reproduce the mode shapes from a small number of known values.

Comparisons between the predictive capabilities of the various methods may be made in quantifiable terms by referring to Table 2, which lists the maximum and average errors produced by each method, normalized by the spacing 


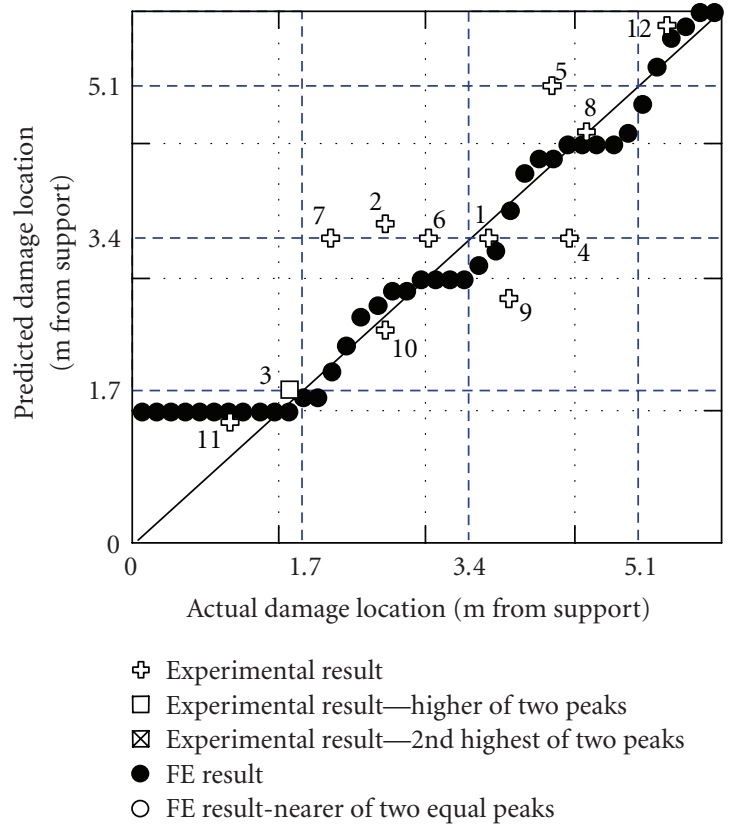

(a) CMS

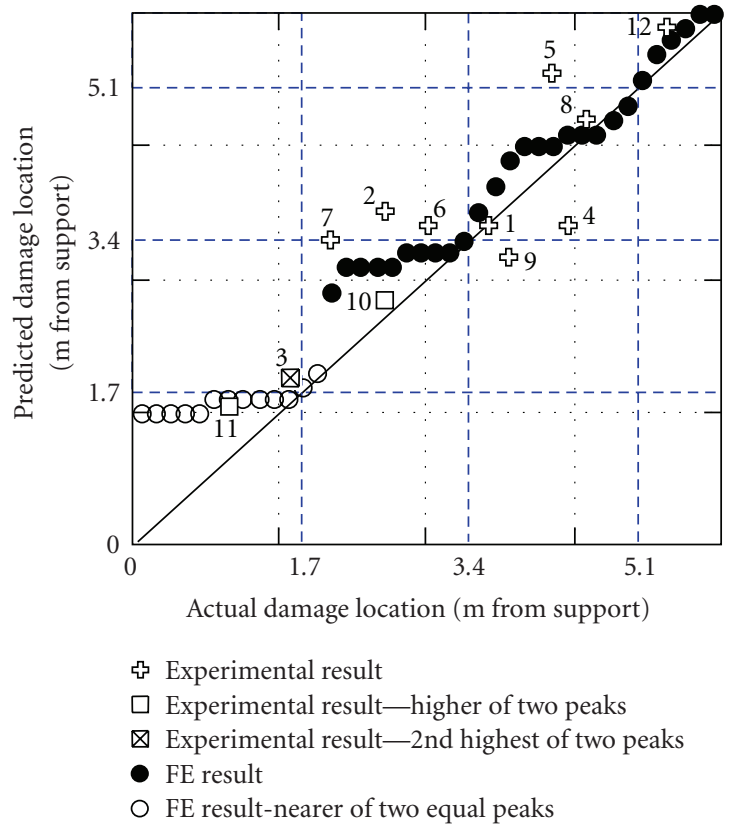

(b) MCF

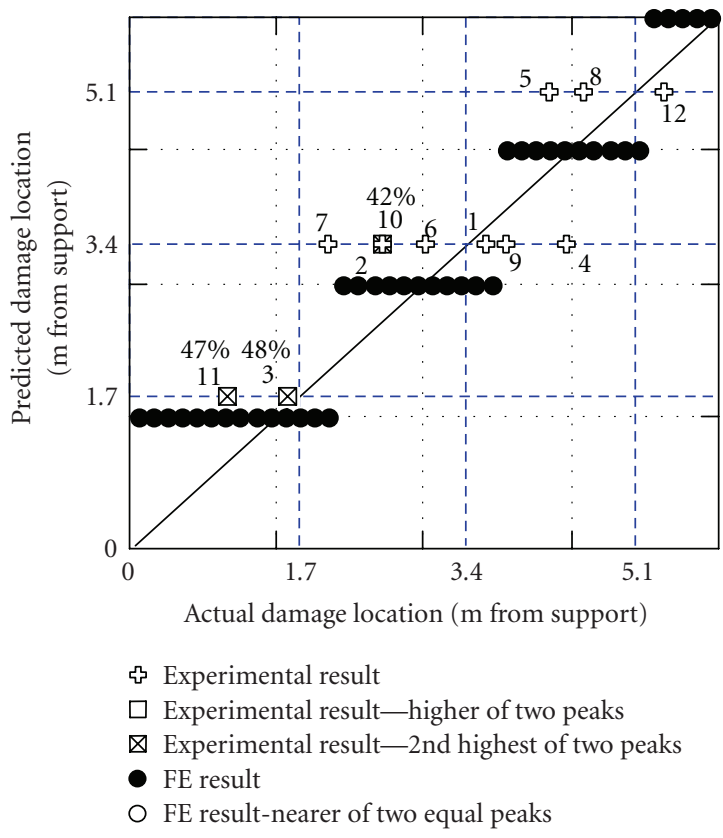

(c) DI

FIGURE 7: Correlation between predicted and actual longitudinal location of damage using six experimental measurement points and seven numerical measurement points, calculated by (a) CMS, (b) MCF, and (c) DI methods. Experimental data points are numbered according to the damage cases given in Figure 4.

TABLE 2: Error in predicted damage location by each method, listed as a fraction of measurement point spacing.

\begin{tabular}{lcccccccc}
\hline \multirow{2}{*}{ Method } & \multicolumn{3}{c}{ Experimental Results } & \multicolumn{3}{c}{ Numerical Results } \\
& Max. error & Avg. error & Stand. dev. & \% ambig* & Max. error & Avg. error & Stand. dev. & \% ambig* \\
\hline CMS & 0.82 & 0.34 & 0.27 & 8 & 0.25 & 0.12 & 0.07 & 0 \\
DI & 0.82 & 0.37 & 0.22 & 25 & 0.55 & 0.25 & 0.15 \\
MCF & 0.82 & 0.35 & 0.26 & 25 & 0.65 & 0.20 & 0.17 \\
\hline
\end{tabular}

* indicates the percentage of damage cases that produced a second peak that was either at least $75 \%$ of the magnitude of the highest peak, or nearer to the damage location. 


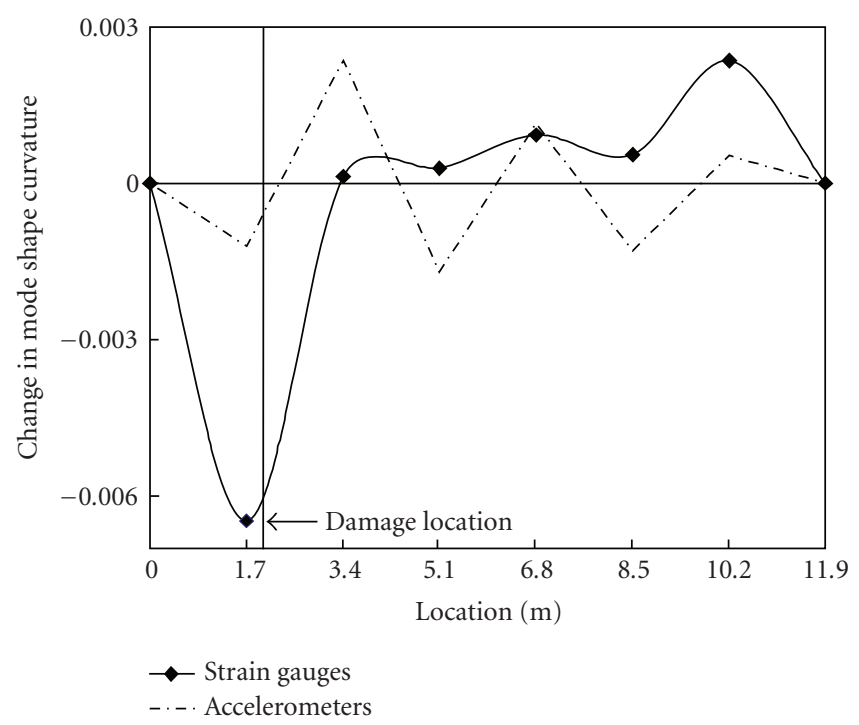

(a)

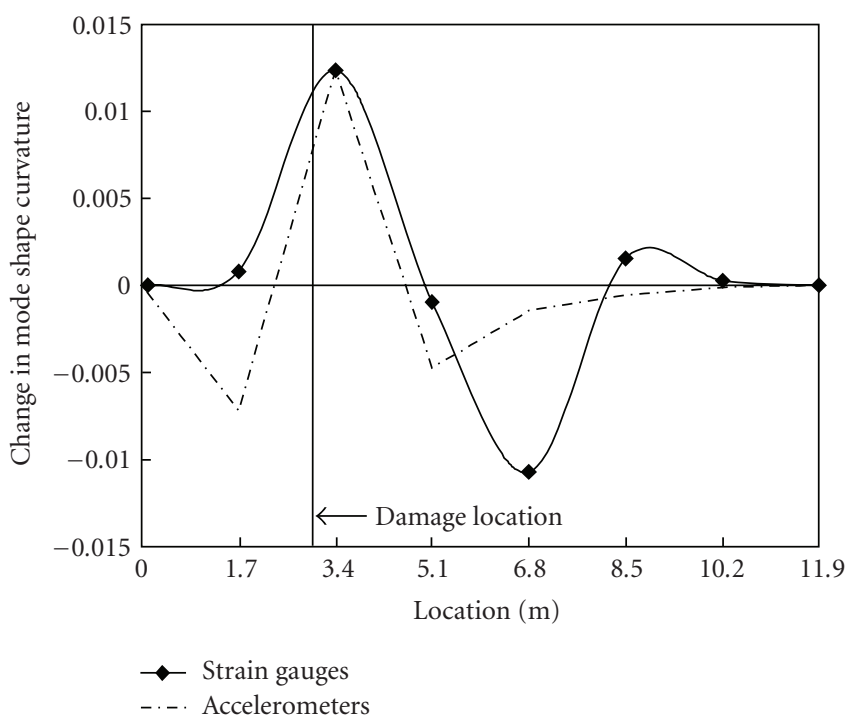

(b)

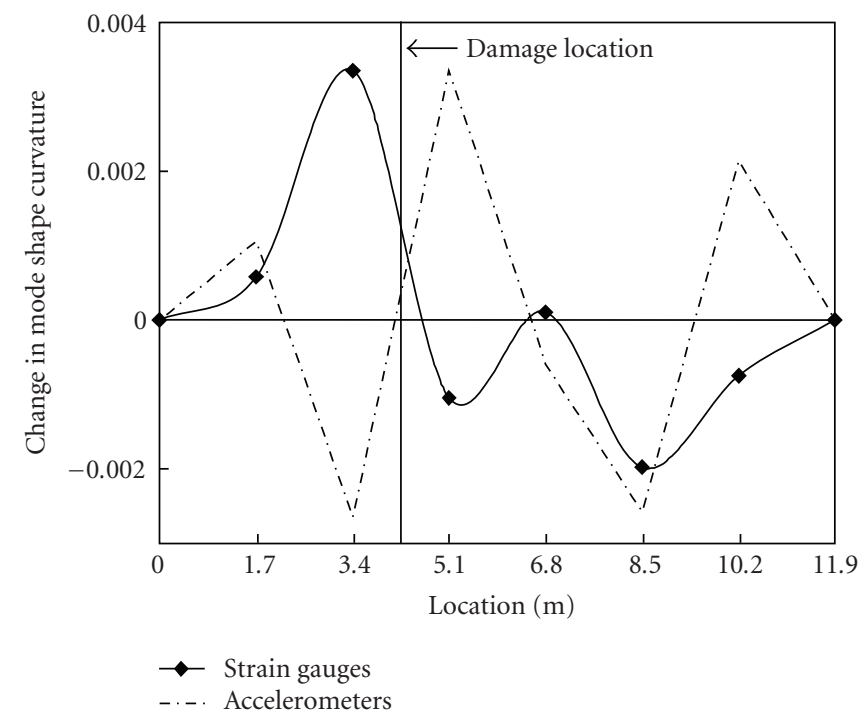

(c)

FIGURE 8: Comparison of MSC distributions produced using strain gauge measured curvatures and those derived from accelerometer data for experimental damage cases (a) 7, (b) 6, and (c) 5 .

between measurement points, $h$, along with the standard deviation of the errors, provided here as an indication of the variability of the predictions. Also listed is the percentage of cases resulting in some ambiguity in the results, as defined earlier. Values corresponding to the numerical results in the table were calculated considering only damage cases located beyond the first measurement point from the support, since the results shown in Figure 7 suggest that damage cases closer to the support require special consideration.

The superior performance of the CMS method relative to other procedures is clearly evident in Table 2 . Based on numerical results, the maximum error in the location of damage predicted by the CMS method was $0.25 h$, with an average error of $0.12 h$. This result was significantly better than the other methods. The advantage diminishes, however, when experimental data are considered, for which all methods produced maximum errors on the order of $0.82 h$ and average errors close to $0.35 h$. However, with one exception, the CMS method always produced a clear maximum peak near the location of damage, thus precluding the possibility of uncertainty with regard to the predicted damage location.

The results presented in Figure 7 and Table 2 suggest that an analyst without any prior knowledge of the damage location would be successful at localizing the damage by interrogating a region within $0.82 h$ on either side of the predicted location when an unambiguous peak appears in the VBDD parameter distributions. As discussed earlier, 
if multiple peaks of similar magnitude appear, in which case the VBDD parameters may differ in the location of the maximum peak, the damage will be located near a support, and the CMS method can be used to localize it. In this case, interrogating a region within approximately $1.5 h$ of the support would be successful at localizing the damage.

Based on these results, it is safe to conclude that the use of a relatively small number of measurement points to characterize the fundamental mode shape is sufficient to detect and localize low-intensity damage with a reasonable level of accuracy for simply supported beam-like structures.

5.2. Direct Measurement of Curvature Using Strain Gauge Clusters. The direct measurement of curvature using vertically aligned strain gauge clusters offers a number of potential advantages over using accelerometers when applying the mode shape curvature method. These include reducing instrumentation costs and increasing the conduciveness to permanent installation of the instrumentation. To facilitate the comparison of the two approaches, the MSC distributions for three representative damage cases are shown in Figure 8, presented in order of increasing distance from the support. Distributions derived from accelerometer data have been scaled such that the maximum values of the two distributions in each graph are identical.

As typical of near support damage cases, Figure 8(a) shows the distributions for experimental damage case 7 , located $2.0 \mathrm{~m}$ from the east (left) support. The large negative peak observed near the damage location in this figure should not be construed as a possible indicator of damage, since this would imply that a local reduction in stiffness has resulted in a decrease in curvature at that location, which would contradict beam theory. For this damage case, then, the strain gauge approach performed very poorly compared to the accelerometer based approach, resulting in a misleading distribution that would lead one to believe that damage was actually located close to the opposite support. A similar result was realized for damage case 11 (not shown), located $1.0 \mathrm{~m}$ from the same support, although the negative peak was absent in this case.

Figure 8(b) shows the distributions for damage case 6, located $3.0 \mathrm{~m}$ from the east (left) support, and may be considered to be representative of damage cases located outside of the near support region. In this case, the two approaches yielded very similar distributions, predicting damage to lie at identical locations. Seven of the 12 damage cases fell into this category. Damage case 3 is included in this group, although it was located only $1.6 \mathrm{~m}$ from the west (right) support (closer to a support than damage case 7 , shown in Figure 8(a)). This lends further support to the contention that support conditions have some influence over the near support distance within which distributions become more difficult to interpret. Uneven support conditions also appear to influence the two approaches somewhat differently. Whereas the accelerometer approach produced multiple peaks in near support damage cases, one of which was typically located near to the damage, the strain gauge approach

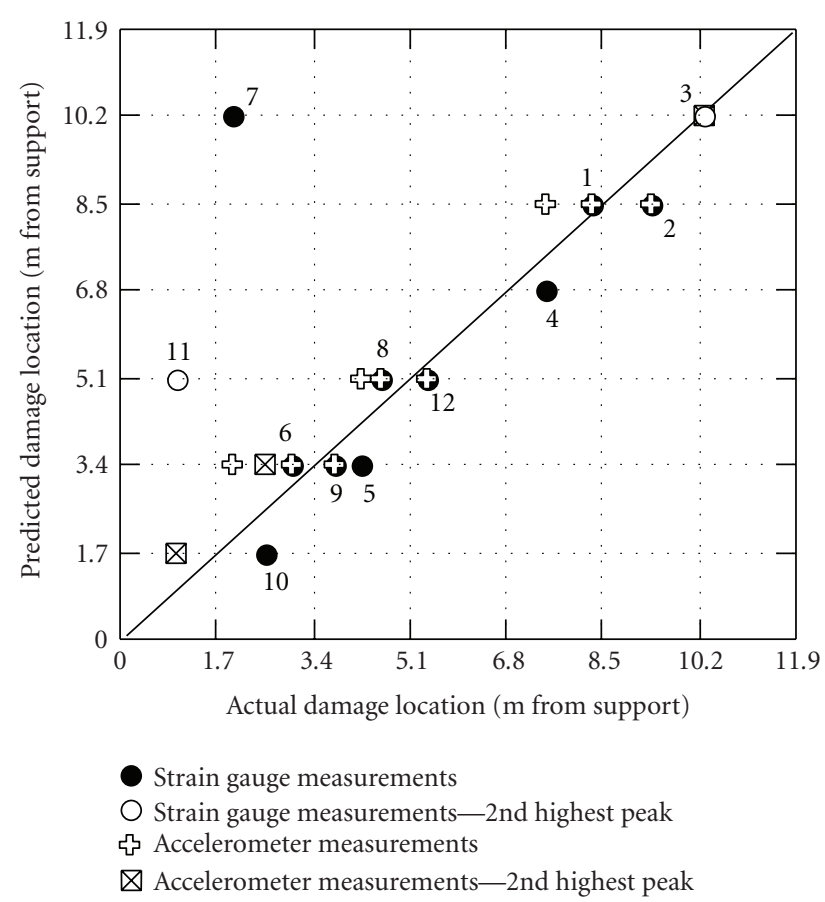

Figure 9: Correlation between predicted and actual damage locations, as calculated by the MSCmethod using strain gauge measured curvatures and those derived from accelerometer data. Numbers refer to experimental damage cases (see Figure 4).

produced distributions in which the most significant peaks were located some distance from the damage.

In two cases (damage cases 4 and 5), for which damage was located midway between two measurement points, the maximum peaks produced by the two approaches appeared on opposite sides of the damage, as seen in Figure 8(c). Such a result could be advantageous if the two instrumentation schemes are used simultaneously, because it would narrow the predicted damage location to lie between the two peaks.

The correlation between predicted and actual damage locations produced by the two approaches for all experimental damage cases is shown in Figure 9. The performance of the two approaches is seen to be generally comparable, with the obvious exception of the two near support damage cases 7 and 11, for which the strain gauge approach produced erroneous results. If these two cases are omitted, the strain gauge approach resulted in maximum and average localization errors of $0.50 h$ and $0.30 h$, respectively, which is an improvement over the MSC method using the accelerometer approach $(0.82 h$ and $0.37 h$, resp., identical to the DI method listed in Table 2). However, the strain gauge approach appears to be more susceptible to producing erroneous or ambiguous results for near support damage cases, perhaps because of lower signal-to-noise ratios near simple supports and the possibility of poor seating at the supports. Therefore, while the MSC method applied using strain gauge data could be used to supplement the other approaches, it should not be relied upon as a stand-alone method. 


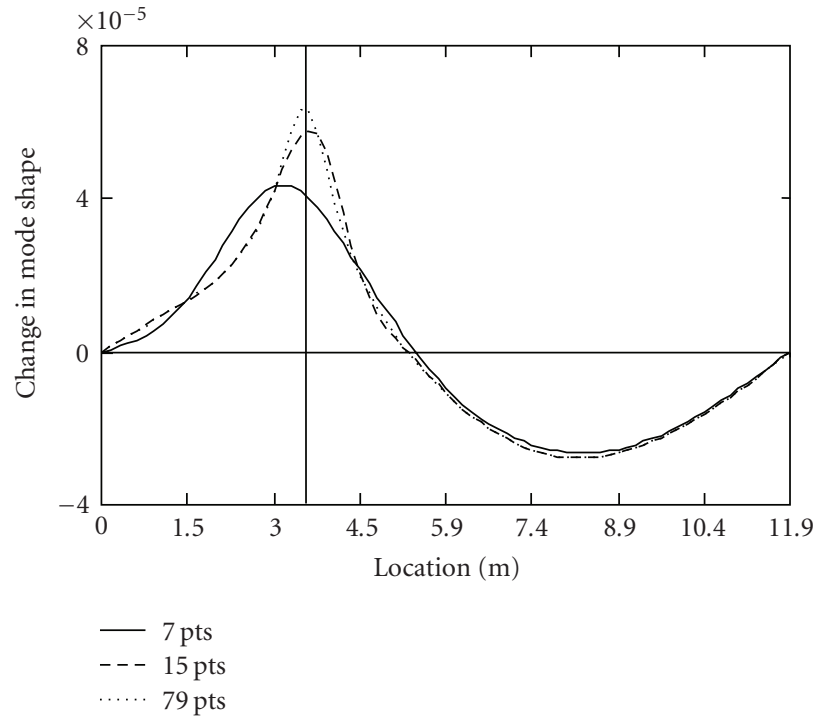

(a)

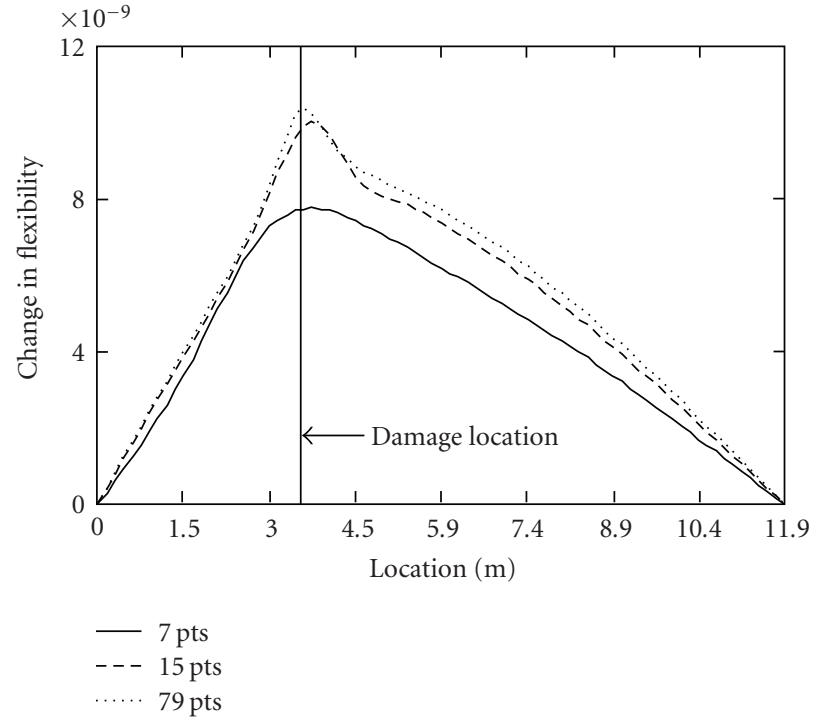

(b)

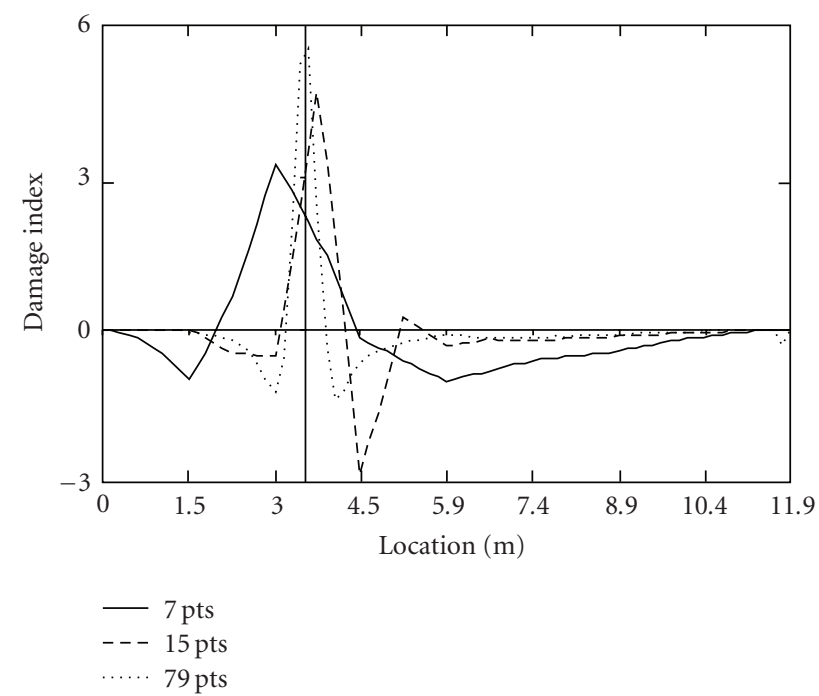

(c)

FiguRE 10: Comparison of VBDD parameter distributions for numerical damage case 24, obtained using 7, 15, and 79 measurement points for (a) the CMS, (b) MCF, and (c) DI methods. Labels along the location axis correspond to the seven measurement points.

5.3. Influence of the Number of Measurement Points. Figure 10 compares the VBDD parameter distributions obtained using an increasing number of $\mathrm{FE}$ simulated measurement points to define the fundamental mode shape for numerical damage case 24 (damage located $3.50 \mathrm{~m}$ from the support, as shown in Figure 4). As the number of measurement points increased, more distinct and narrower peaks were produced, particularly by the DI method; in addition, the peaks were located closer to the damage location. Improved predictive capability was therefore achieved by using a larger number of measurement points.

A similar trend was observed when damage was located nearer to a support, as shown in Figure 11 (corresponding to numerical damage case 7 , located $0.97 \mathrm{~m}$ from a support). In this case, increasing the number of measurement points effectively removed additional peaks produced by the DI method when a small number of points were used. However, the second broad hump produced by the MCF method was not affected by increasing the number of measurement points. This second hump is therefore not a direct result of the use of an inadequate number of measurement points. In fact, it can be demonstrated that this feature is characteristic of the MCF method for low level damage located near the support. It indicates that the maximum increase in deflection along the beam caused by inducing damage near the support is roughly the same whether a unit load is applied at the damage location or near midspan. 
TABLE 3: Error in predicted damage location by each method, listed as a fraction of measurement point spacing, when 15 and 79 measurement points were used.

\begin{tabular}{lcccccccc}
\hline \multirow{2}{*}{ Method } & \multicolumn{9}{c}{15 points } & \multicolumn{3}{c}{79 points } \\
& Max. error & Avg. error & Stand. dev. & \% ambig* & Max. error & Avg. error & Stand. dev. & $\%$ ambig* \\
\hline CMS & 0.30 & 0.12 & 0.06 & 0 & 0.50 & 0.50 & 0.003 & 0 \\
DI & 0.50 & 0.26 & 0.15 & 0 & 0.50 & 0.50 & 0.001 \\
MCF & 0.50 & 0.20 & 0.14 & 40 & 0.50 & 0.50 & 0.001 & 4.5 \\
\hline
\end{tabular}

* indicates the percentage of the 40 cases that produced a second peak that was either at least $75 \%$ of the magnitude of the highest peak, or nearer to the damage location, including near support damage cases.

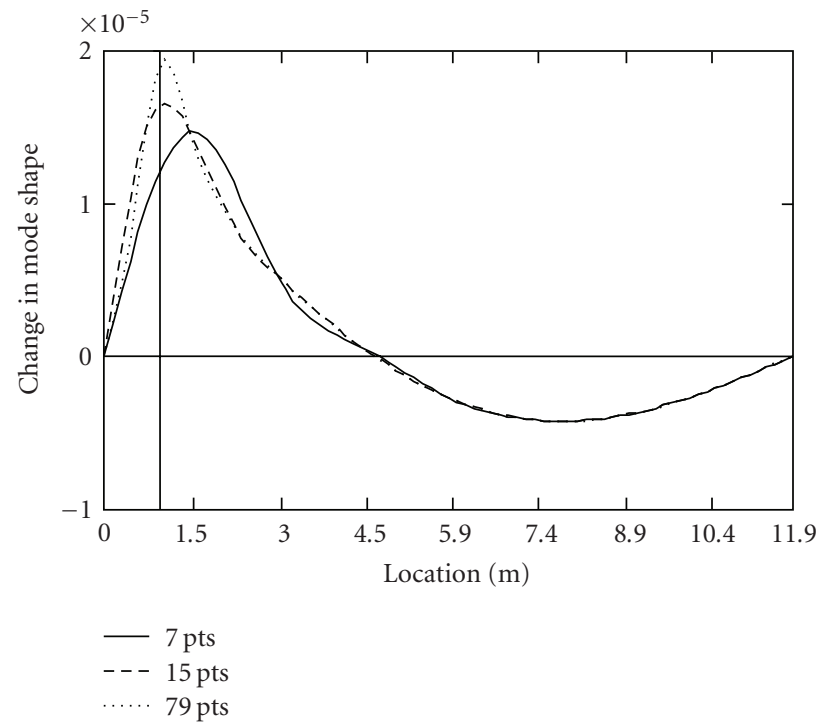

(a)

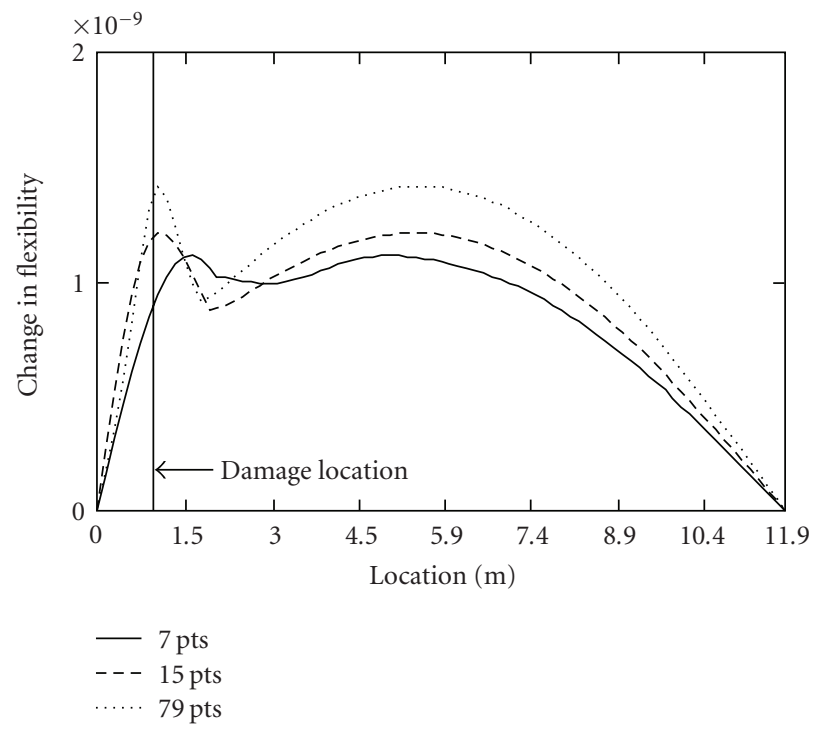

(b)

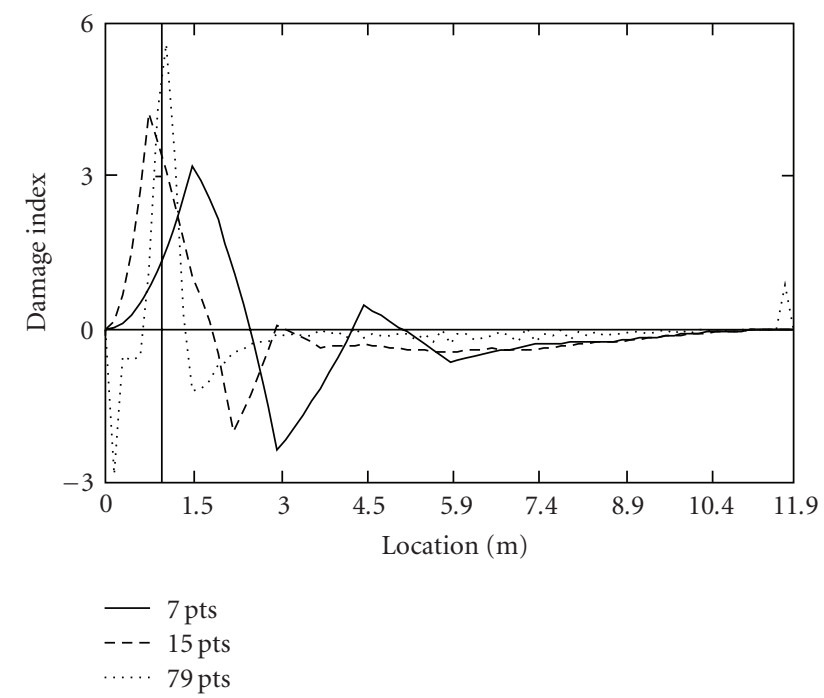

(c)

Figure 11: Comparison of VBDD parameter distributions for numerical damage case 7, obtained using 7, 15, and 79 measurement points for (a) the CMS, (b) MCF, and (c) DI methods. Labels along the location axis correspond to the seven measurement points.

The performance using 15 and 79 measurement points more generally is illustrated in Figure 12, in which correlations between predicted and actual locations of damage are plotted for the CMS and DI methods. The MCF method produced results similar to the CMS method, although with greater ambiguity near the support. The improvement in localization accuracy with an increasing number of measurement points is clearly evident, with very well-defined mode shapes allowing damage to be localized with great accuracy, regardless of the method used. Although implementing 


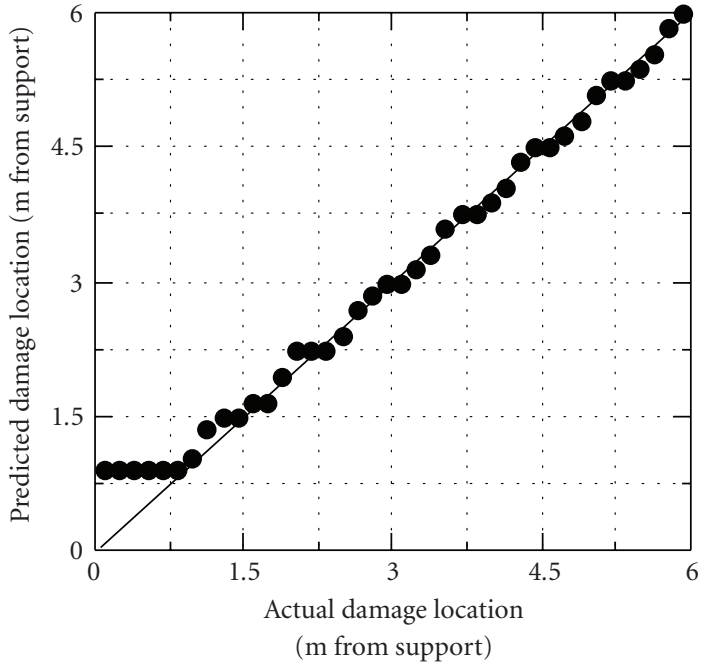

(a) CMS 15 pts

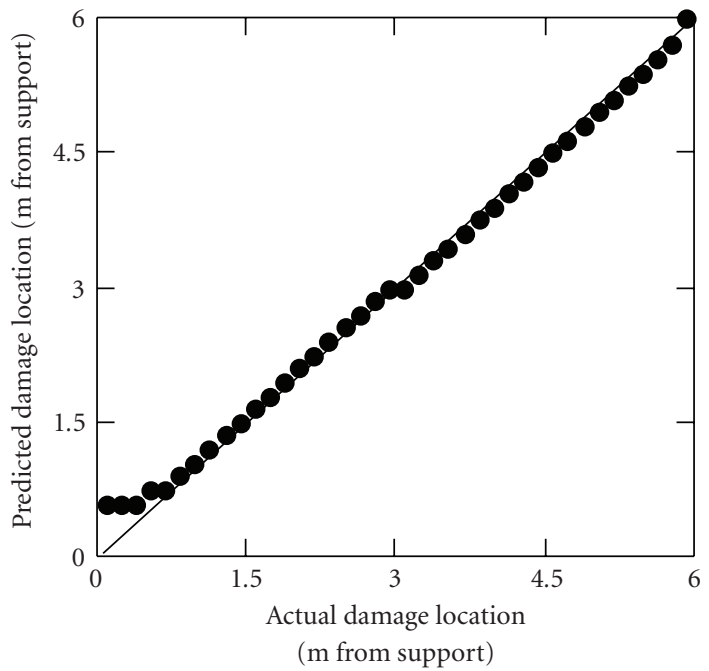

(c) CMS 79 pts

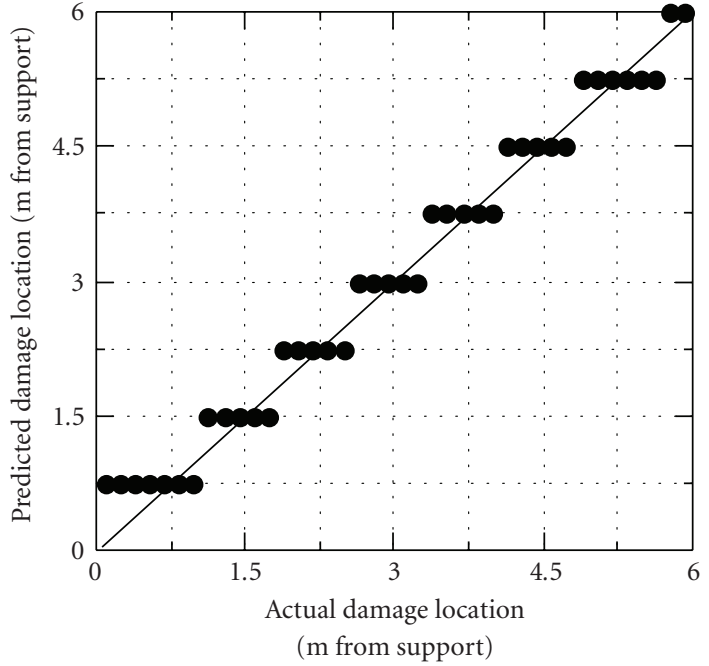

(b) DI 15 pts

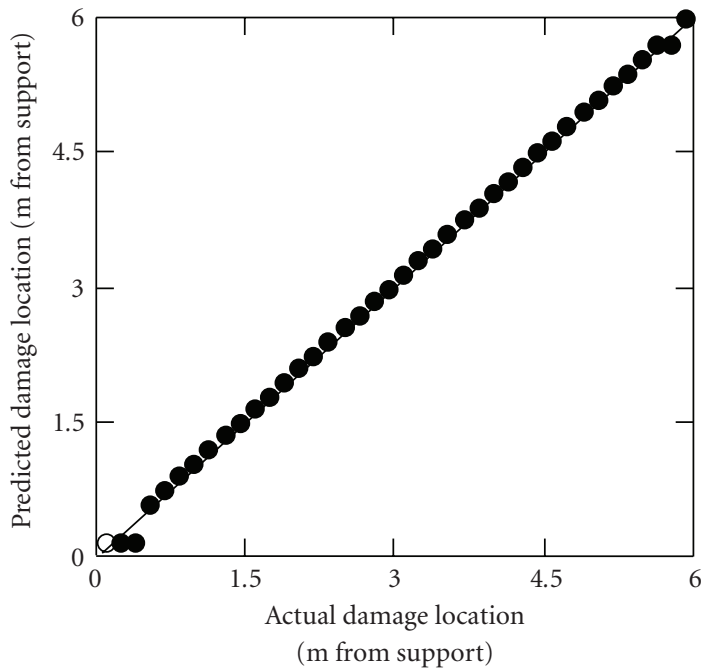

(d) DI 79 pts

FIGURE 12: Correlation between predicted and actual damage locations, calculated using 15 measurement points by (a) the CMS and (b) the DI methods; and calculated using 79 measurement points by (c) the CMS and (d) the DI methods.

an experimental system to achieve this level of definition may be impractical, the excellent performance observed in Figures 12(c) and 12(d) confirms the soundness of the theory underlying VBDD and its applicability to a simply supported beam-like component.

The maximum and average errors produced by all methods, normalized by measurement point spacing, are summarized in Table 3. In relative terms, these results are seen to be very similar to those achieved using seven measurement points, as listed in Table 2. Localization accuracy is therefore directly proportional to measurement point spacing, $h$, with maximum errors in the order of $0.5 h$ and average errors typically between $0.20 h$ and $0.26 h$ when seven or 15 measurement points were used. The CMS method performed somewhat better, but, as was previously mentioned, this improvement was not observed when experimental data were used.
5.4. Use of Additional Modes. Notwithstanding the excellent performance of the techniques using only the fundamental mode, perhaps leaving little room for expected improvement, the influence of including additional modes was also investigated. Conceptually, the use of additional modes might be expected to improve the performance of the VBDD methods by virtue of the fact that additional information is provided and that higher flexural modes should be more sensitive to local changes in stiffness. To investigate this hypothesis, the first three FE generated flexural modes (modes 1, 2, and 4 in Table 1) were incorporated into the methods.

The CMS was first calculated using each mode individually. Figure 13(a) shows the resulting distributions for numerical damage case 24 , located $3.50 \mathrm{~m}$ from the support, when seven measurement points were used. When examined together, the set of three distributions provides a clear 


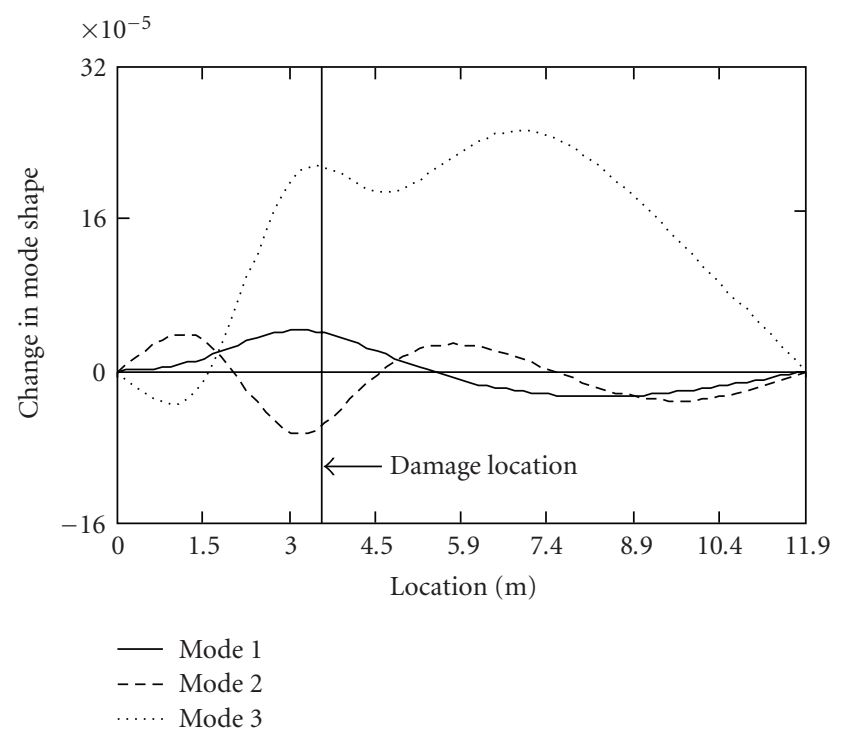

(a) $7 \mathrm{pts}$

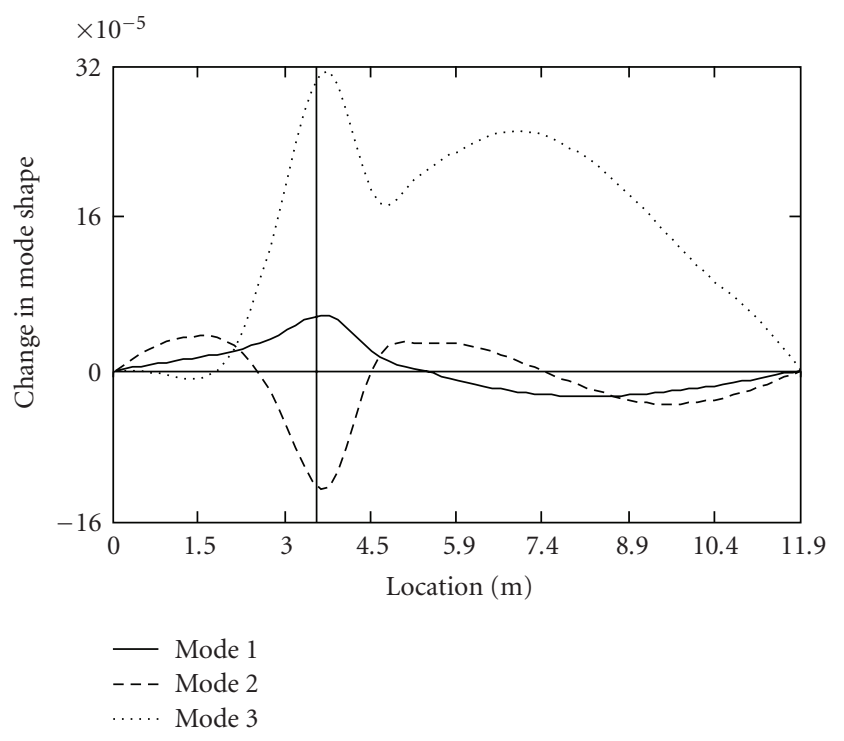

(b) $15 \mathrm{pts}$

FIGURE 13: Changes in the first three flexural mode shapes caused by numerical damage case 24 , located $3.50 \mathrm{~m}$ from the support, calculated using (a) 7 and (b) 15 simulated measurement points.

TABLE 4: Error in predicted damage location by each method, listed as a fraction of measurement point spacing, when two and three modes were used in combination with 7 measurement points.

\begin{tabular}{lcccccccc}
\hline \multirow{2}{*}{ Method } & \multicolumn{4}{c}{ 2 modes } & \multicolumn{3}{c}{3 modes } \\
& Max. error & Avg. error & Stand. dev. & \% ambig* & Max. error & Avg. error & Stand. dev. & $\%$ ambig* \\
\hline CMS & 0.35 & 0.15 & 0.08 & 2.5 & 0.25 & 0.14 & 0.07 & 5 \\
DI & 0.95 & 0.33 & 0.24 & 2.5 & 0.65 & 0.26 & 0.18 \\
MCF & 0.45 & 0.18 & 0.13 & 0 & 0.45 & 0.16 & 0.11 & 7.5 \\
\hline
\end{tabular}

* indicates the percentage of the 40 cases that produced a second peak that was either at least $75 \%$ of the magnitude of the highest peak, or nearer to the damage location, including near support damage cases.

indication of the location of the damage. Each distribution features a peak near the damage location, although it is not necessarily positive (mode 2 ) or the highest peak (mode 3 ). The latter difficulty was removed when mode shapes were defined by a larger number of measurement points (Figure 13(b)), but a significant second peak still remained in the change of the third mode shape. In addition, the magnitude of the peaks produced by different modes differed significantly.

In order to combine the three individual mode shape changes into a single distribution representative of the changes in all three mode shapes, the absolute value of each individual distribution was first calculated, the result was normalized by its root-mean-square (rms) value to remove the influence of magnitude differences, and then the three distributions were added together. Results of this approach are presented together with those of the other VBDD methods in the following paragraphs, with a focus on results achieved using seven measurement points.

Figure 14 shows how the distributions for the three methods changed as an increasing number of modes were used. This figure corresponds again to numerical damage case 24 and the use of seven measurement points. Very little change in either the clarity or accuracy of the damage localization is observed as the number of modes increases, although one could argue that some improvement in clarity is apparent with the CMS method (Figure 14(a)). Similar results were observed for near support damage cases. The only improvement of significance occurred when the MCF method was used for near support damage cases (see Figure 15(a)). In these cases, the ambiguity caused by the occurrence of a second broader peak was removed when higher modes were considered. Interestingly, this improvement was not as significant when mode shapes were more well-defined using 15 measurement points, as seen in Figure 15(b), implying that the improvement for seven measurement points was artificial.

The performance of the methods more generally as the number of modes increased is shown in Table 4, in which the maximum and average localization errors for all 40 numerical damage cases are listed, along with the percentage of cases resulting in some ambiguity, when seven measurement points were used. Considering localization accuracy and the number of ambiguities produced, comparisons both within Table 4 and to Table 2 show that either no improvement was achieved with an increase in the number of 

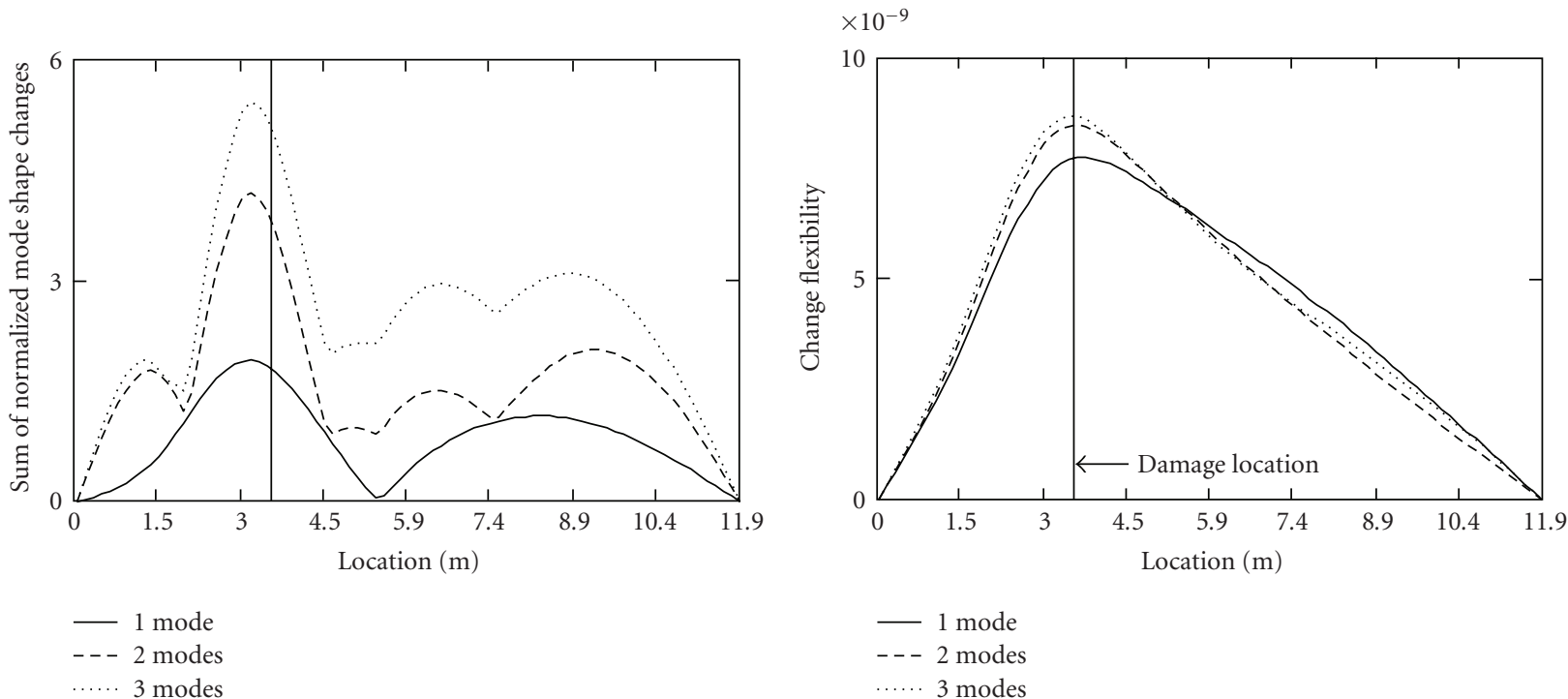

(a)

(b)

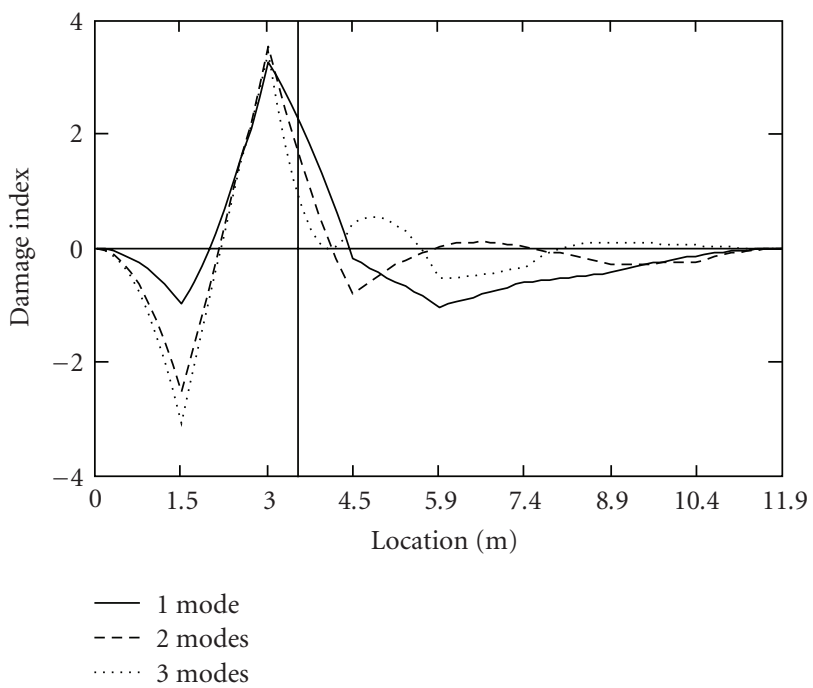

(c)

FIGURE 14: VBDD distributions calculated using the first three flexural modes and 7 measurement points for numerical damage case 24 , located $3.50 \mathrm{~m}$ from the support, by the (a) CMS, (b) MCF, and (c) DI methods.

modes considered, or that a decline in performance resulted. The only exception to this trend was observed with the MCF method, which showed improvements in both criteria. In particular, increasing the number of modes effected a reduction in the ambiguities observed by the MCF method in near support damage cases. The decline in performance of the DI method was seen to be associated primarily with damage cases located near a node point for a particular mode shape. A similar decline was not observed for the other two curvature-based methods (MSC and CUFC methods).

Table 5 provides an indication of the performance as the number of modes increased when 15 measurement points were used to define the mode shapes. Comparison with Table 3 shows that the use of two or three modes produced either no improvement or a decline in performance relative to the use of only the fundamental mode. Comparison with Table 4 shows that, in relative terms, the use of 15 points in combination with two or three modes produced marginal localization improvement only for the DI method, compared to seven measurement points. A decline was observed for other two methods, although localization was still observed to be more or less proportional to the spacing between measurement points.

Thus, any improvements associated with the use of higher modes were minimal and restricted to only one of the methods. Declines in performance were also observed. These results are not surprising. The excellent performance of the VBDD methods when using only the fundamental mode leaves very little room for improvement, given the theoretical limitations of the methods. Although not considered here, 


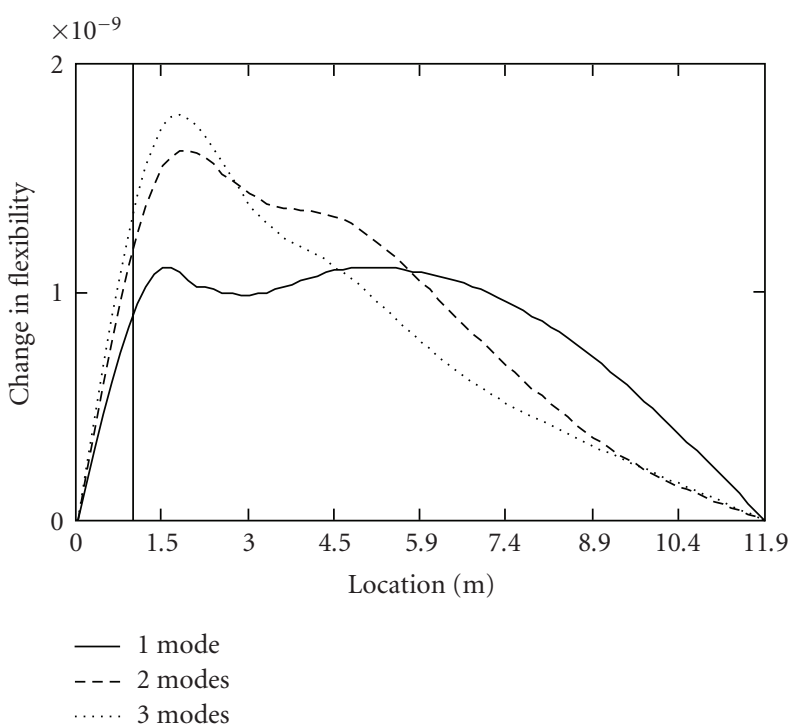

(a)

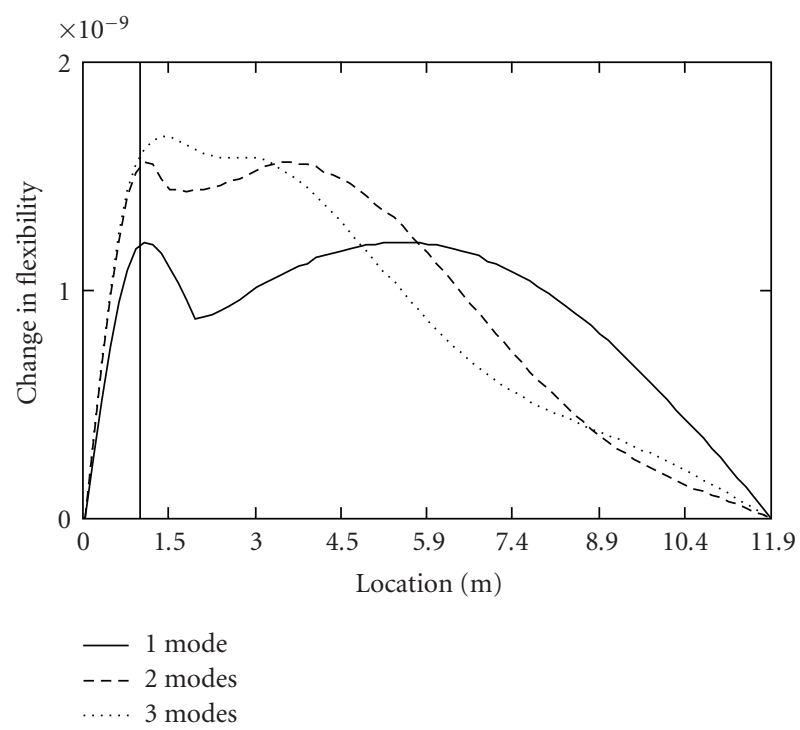

(b)

FIGURE 15: Change in flexibility calculated using the first three flexural modes for numerical damage case 7 , located $0.97 \mathrm{~m}$ from the support, using (a) 7 and (b) 15 simulated measurement points.

TABLE 5: Error in predicted damage location by each method, listed as a fraction of measurement point spacing, when two and three modes were used in combination with 15 measurement points.

\begin{tabular}{lcccccccc}
\hline \multirow{2}{*}{ Method } & \multicolumn{4}{c}{2 modes } & \multicolumn{3}{c}{3 modes } \\
& Max. error & Avg. error & Stand. dev. & \% ambig* & Max. error & Avg. error & Stand. dev. & $\%$ ambig* \\
\hline CMS & 0.50 & 0.14 & 0.09 & 2.5 & 0.50 & 0.14 & 0.09 & 0 \\
DI & 0.70 & 0.28 & 0.17 & 2.5 & 0.50 & 0.25 & 0.15 \\
MCF & 0.70 & 0.20 & 0.15 & 27.5 & 0.50 & 0.19 & 0.13 \\
\hline
\end{tabular}

${ }^{*}$ indicates the percentage of the 40 cases that produced a second peak that was either at least $75 \%$ of the magnitude of the highest peak, or nearer to the damage location, including near support damage cases.

it should also be noted that, in practice, measurements of higher modes are generally subject to lower signalto-noise ratios, since modal amplitudes tend to diminish with higher modes, leading to additional disincentive for using higher modes. To summarize, the minimal benefit and increased uncertainty associated with the use of higher modes provides a compelling argument in favour of using only the fundamental mode when applying the VBDD methods considered in this study.

5.5. Summary. Table 6 summarizes the results of this study, highlighting the influence of the factors investigated. The CMS method, applied using only the fundamental mode and a relatively small number of measurement points, appears to be the most attractive alternative.

\section{Conclusions}

This study has shown that damage on the surface of a fullscale precast, prestressed concrete box girder, corresponding to a local reduction in stiffness of only $2.5 \%$, can be detected using VBDD techniques and localized to a region within approximately 1.6 times the longitudinal spacing between uniformly distributed sensors, $h$. This was achieved using data for only the fundamental mode shape before and after damage, defined by as few as six evenly spaced measurement points. In contrast to the clear and unambiguous peaks generally observed in the VBDD distributions near the location of damage, damage cases located closer to supports resulted in VBDD distributions featuring multiple peaks and undulations. In these cases, damage could be localized to a region within approximately $1.5 \mathrm{~h}$ from the support, although this "near support" region depended to some extent on support conditions.

While any of the VBDD methods considered was shown to be capable of detecting and localizing the majority of damage cases investigated, the CMS method provided the clearest indication of damage over the widest range of damage locations, particularly for near support damage cases. The CF method, modified slightly from its original formulation for improved performance, was less clear in its indication of near support damage cases. The DI method (as well as the other two curvature-based methods) tended to produce 


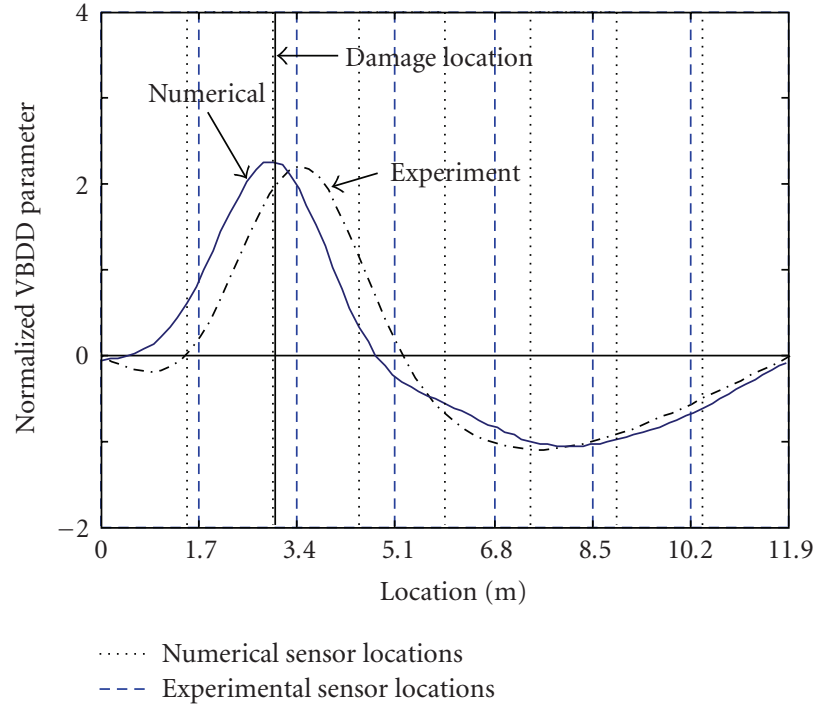

(a)

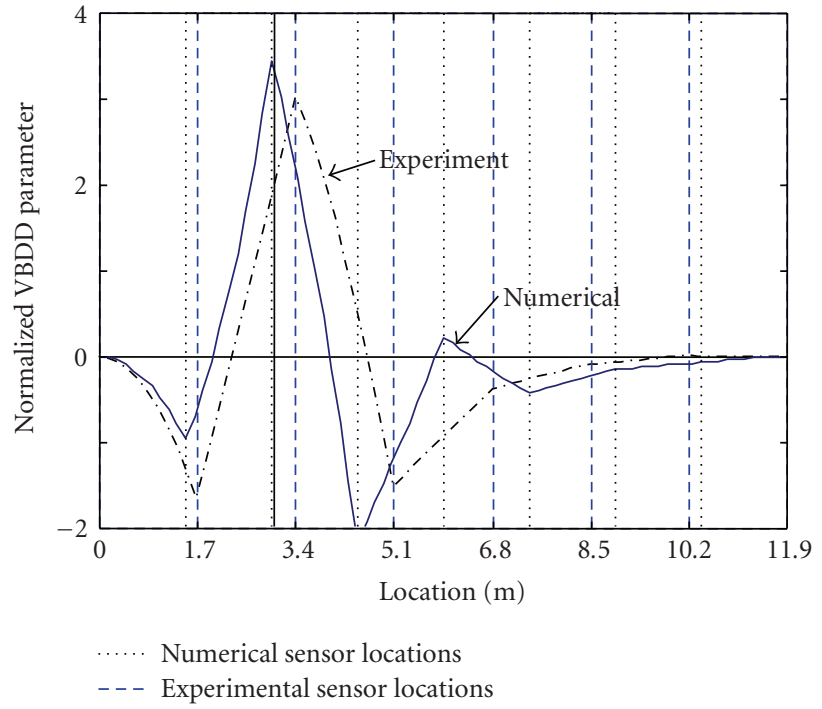

(b)

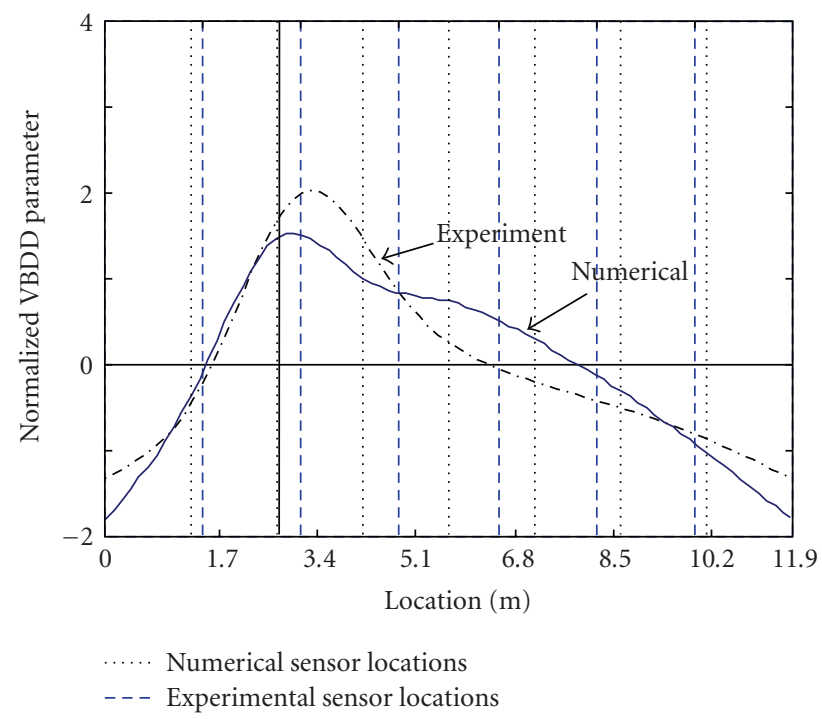

(c)

FIGURE 16: Comparison of VBDD parameter distributions produced for experimental damage case 6 (3.0 m from support, 6 measurement points) and numerical damage case 21 (3.05 m from support, 7 simulated measurement points) by (a) CMS, (b) DI, and (d) MCF methods.

several ambiguous indications of damage. Results indicated that the simultaneous use of several VBDD methods could provide an added level of confidence when localizing the damage, but that the CMS method was the most reliable.

In comparison to the use of accelerometers mounted on the top surface of the girder, application of the MSC method using data from strain gauge clusters configured to measure curvature directly was found to perform slightly better, as long as damage was not located too near a support (e.g., within approximately $1.5 h$ of the support). In those cases, very misleading predictions sometimes resulted. Consequently, the use of strain gauge data alone is a less attractive alternative.
The accuracy of damage localization was directly proportional to the spacing between measurement points. Increasing the number of measurement points will therefore lead to a proportional increase in localization accuracy. All of the VBDD methods investigated were found to be extremely effective at pinpointing the location of damage when mode shapes were very well-defined with a large number of measurement points. Notwithstanding the challenges associated with achieving this level of mode shape definition in practice, this result confirms the soundness of VBDD theory and its usefulness for damage localization.

The use of two additional flexural modes, in addition to the fundamental vibration mode, did not generally improve 
TABLE 6: Summary of performance of the three VBDD methods and the influence of the factors studied.

\begin{tabular}{|c|c|c|c|}
\hline Factors & CMS & MCF & DI \\
\hline $\begin{array}{l}\text { Reference conditions: } \\
\text { (i) } 7 \text { meas. points } \\
\text { (ii) Fundamental mode } \\
\end{array}$ & $\begin{array}{l}\text { Unambiguous indication of } \\
\text { damage location within } \\
\pm 0.8 \mathrm{~h}\end{array}$ & $\begin{array}{l}\text { Unambiguous indication of } \\
\text { damage location within } \\
\pm 0.8 \mathrm{~h}\end{array}$ & $\begin{array}{l}\text { Unambiguous indication of } \\
\text { damage location within } \\
\pm 0.8 \mathrm{~h}\end{array}$ \\
\hline Near support damage cases & $\begin{array}{l}\text { Able to locate damage with } \\
\text { little ambiguity }\end{array}$ & $\begin{array}{l}\text { Multiple peaks indicating } \\
\text { that damage is located near } \\
\text { a certain support }\end{array}$ & $\begin{array}{l}\text { Misleading predictions of } \\
\text { damage location }\end{array}$ \\
\hline $\begin{array}{l}\text { Use of additional } \\
\text { measurement points }\end{array}$ & $\begin{array}{l}\text { Proportional increase in } \\
\text { localization accuracy }\end{array}$ & $\begin{array}{l}\text { Proportional increase in } \\
\text { localization accuracy }\end{array}$ & $\begin{array}{l}\text { Proportional increase in } \\
\text { localization accuracy }\end{array}$ \\
\hline Use of additional modes & No improvement & $\begin{array}{l}\text { Reduction in ambiguity for } \\
\text { some near support damage } \\
\text { cases }\end{array}$ & $\begin{array}{l}\text { Decline in localization } \\
\text { accuracy }\end{array}$ \\
\hline $\begin{array}{l}\text { Combined use of additional } \\
\text { modes and additional } \\
\text { measurement points }\end{array}$ & No improvement & No improvement & $\begin{array}{l}\text { Some improvement relative } \\
\text { to the use of additional } \\
\text { modes alone }\end{array}$ \\
\hline
\end{tabular}

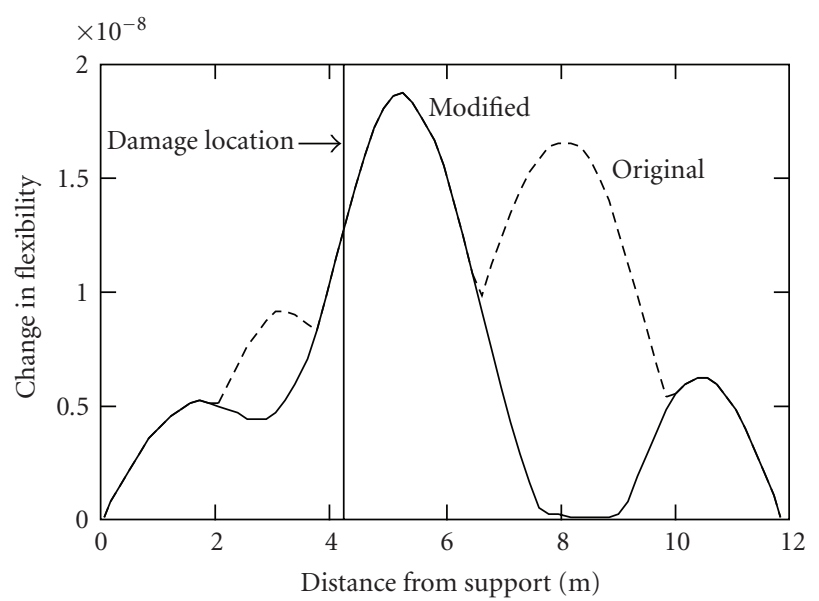

(a)

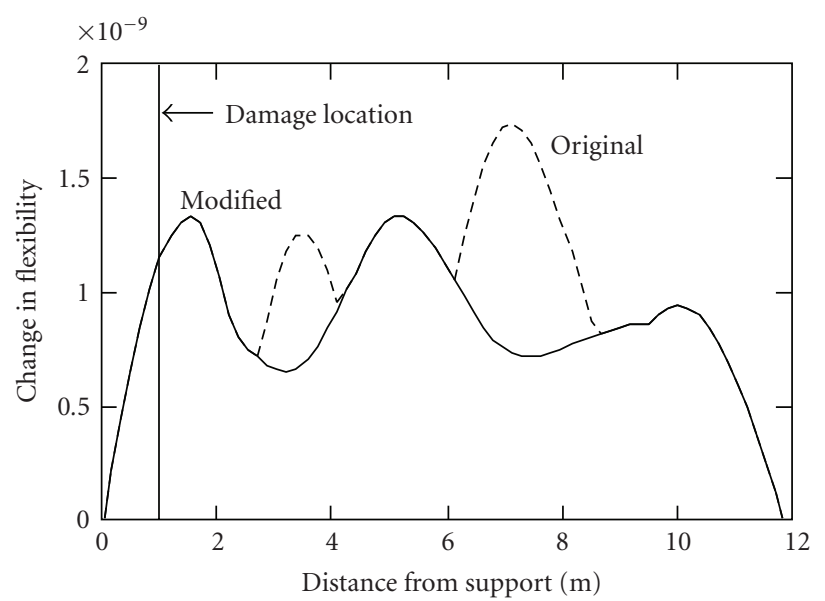

(b)

FIGURE 17: Distribution of change in flexibility by original and modified methods for experimental damage cases (a) 5 and (b) 11 .

the performance of the VBDD techniques investigated. The only advantage appeared to be the removal of ambiguity when the MCF method was used for near support damage cases. For other VBDD methods, the use of two additional modes resulted in either no improvement, or a decline in performance. The use of only the fundamental mode is therefore considered to be sufficient for damage localization.

This study has shown that VBDD methods are effective at detecting and localizing damage on full-scale beam-like bridge elements, such as precast, prestressed concrete box girders, using data that can be obtained relatively easily using a small number of sensors and only the fundamental mode of vibration. Given that large portions of these elements are generally inaccessible to inspection by other SHM techniques, VBDD methods appear to be particularly attractive for the early detection of damage.

\section{Appendices}

\section{A. Validation of Numerical Simulations}

In order to establish the validity of the numerical calculations and justify the extension of the study to a larger number of damage cases using FE simulations, comparison of the numerical and experimental results for a representative damage case is presented here. Figure 16 displays the VBDD distributions for experimental damage case $6(3.0 \mathrm{~m}$ from support) and numerical damage case $21(3.05 \mathrm{~m}$ from support). It should be recalled that six uniformly distributed measurement points were used experimentally, while seven were used numerically, so identical results should not be expected. Experimental measurement locations are indicated by the dashed and labelled gridlines, while numerical measurement points are indicated by additional dotted 


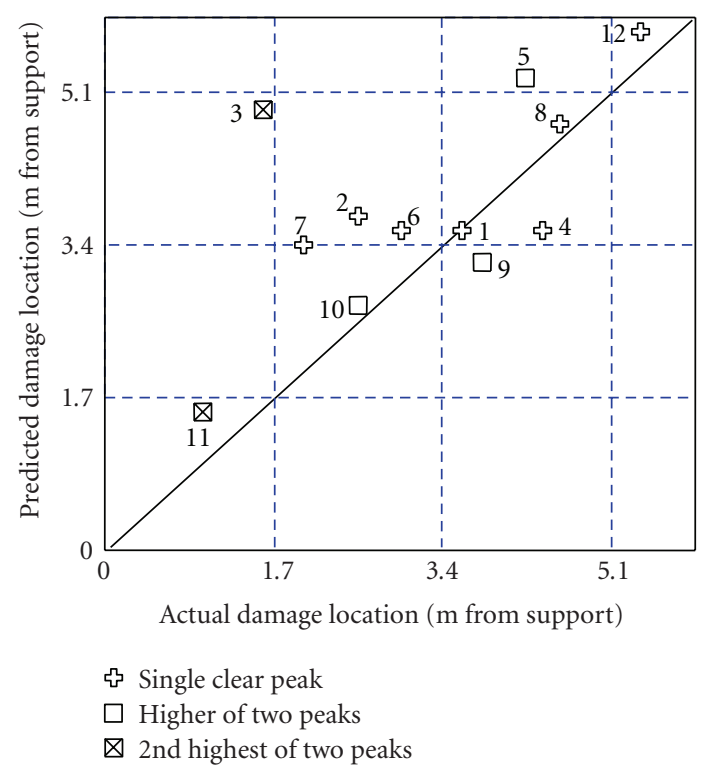

(a) $\mathrm{CF}$

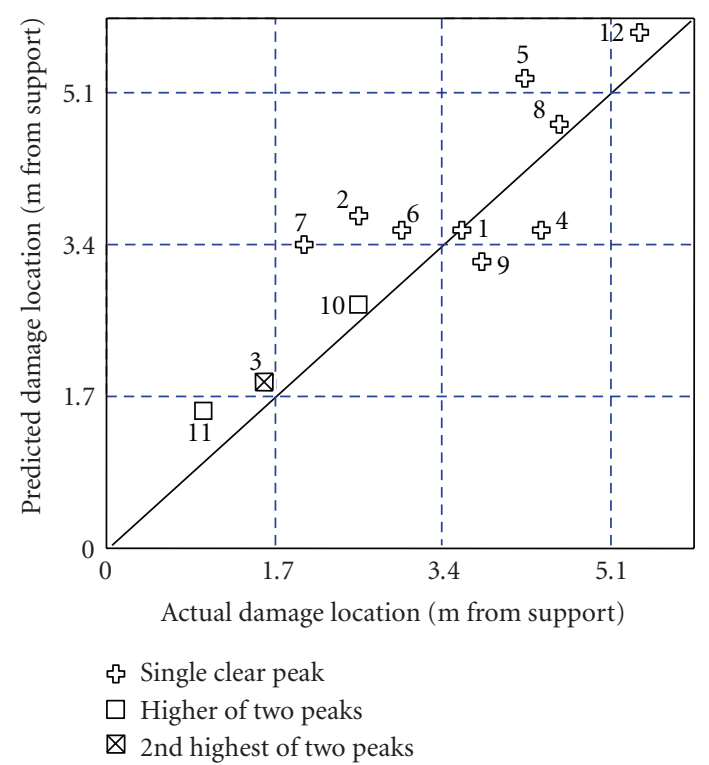

(b) MCF

Figure 18: Correlation between predicted and actual location of experimental damage cases using six measurement points, calculated by the CF method in (a) original and (b) modified forms. Data points are numbered according to the experimental damage cases shown in Figure 4.

gridlines. The similarities between the resulting distributions are clearly evident for all VBDD methods, with each pair of distributions featuring similar characteristics and peaks occurring at the measurement point nearest to the damage location. The use of FE simulations to study a wider distribution of damage cases can therefore be shown to be well-founded.

\section{B. Comparison of Change in Flexibility Method in Original and Modified Forms}

To compare the distributions produced by the change in flexibility method in original and modified forms, Figure 17 presents the two distributions produced for experimental damage cases 5 (within the middle third of the span) and 11 (within $1 \mathrm{~m}$ of the support). As seen, the original and modified distributions are identical except at the dashed lines, where the original method picks up the absolute values of negative changes in flexibility to produce additional peaks. These additional peaks are invariably located farther from the damage than the most significant peak common to both distributions; in addition, some of the additional peaks are also higher in magnitude. As such, an added level of uncertainty is introduced by considering the absolute values, making the distributions more difficult to interpret.

Figure 18 illustrates the resulting localization performance of the two methods for all 12 experimental damage cases. It is evident that the modified method removes several ambiguities (cases 5, 9, and 11) and improves the accuracy of the predicted location (case 3). The modified method was therefore used for the current study. It should be noted, though, that differences between the two methods were only apparent when the experimental data were used. Numerically generated data always resulted in change in flexibility matrices that contained only positive values, as would be expected theoretically.

\section{Acknowledgments}

The financial support of the ISIS Canada Network of Centres of Excellence and the Natural Sciences and Engineering Research Council of Canada is gratefully acknowledged. The authors are also grateful to the Saskatchewan Ministry of Highways and Infrastructure for providing the girder.

\section{References}

[1] Federal Highway Administration, "Reliability of visual inspection," Tech. Rep. FHWA-RD-01-020 and FHWA-RD-01-021, Federal Highway Administration, Washington, DC, USA, 2001.

[2] L. Cartz, Non-Destructive Testing, ASM International, Materials Park, Ohio, USA, 1995.

[3] B. Raj, T. Jayakumar, and M. Thavasimuthu, Practical NonDestructive Testing, Narosa Publishing House, New Delhi, India, 2nd edition, 2002.

[4] C. H. Jenkins, L. Kjerengtroen, and H. Oestensen, "Sensitivity of parameter changes in structural damage detection," Shock and Vibration, vol. 4, no. 1, pp. 27-37, 1997.

[5] J. L. Schulz, B. Commander, G. G. Goble, and D. M. Frangopol, "Efficient field testing and load rating of short- and mediumspan bridges," Structural Engineering Review, vol. 7, no. 3, pp. 181-194, 1995.

[6] S. W. Doebling, C. R. Farrar, M. B. Prime, and D. W. Shevitz, "Damage identification and health monitoring of structural and mechanical systems from changes in their vibration characteristics: a literature review," Tech. Rep. LA 13070-MS, Los Alamos National Laboratory, Los Alamos, NM, USA, 1996. 
[7] Z. Zhou, L. D. Wegner, and B. F. Sparling, "Vibration-based detection of small-scale damage on a bridge deck," Journal of Structural Engineering, vol. 133, no. 9, pp. 1257-1267, 2007.

[8] S. W. Doebling, C. R. Farrar, and M. B. Prime, "A summary review of vibration-based damage identification methods," Shock and Vibration Digest, vol. 30, no. 2, pp. 91-105, 1998.

[9] H. Sohn, C. R. Farrar, F. M. Hemez, D. D. Shunk, D. W. Stinemates, and B. R. Nadler, "A review of structural health monitoring literature: 1996-2001," Tech. Rep. LA-13976-MS, Los Alamos National Laboratory, Los Alamos, NM, USA, 2003.

[10] P. Cawley and R. D. Adams, "The location of defects in structures from measurements of natural frequencies," The Journal of Strain Analysis for Engineering Design, vol. 14, no. 2, pp. 49-57, 1979.

[11] O. S. Salawu, "Detection of structural damage through changes in frequency: a review," Engineering Structures, vol. 19, no. 9, pp. 718-723, 1997.

[12] B. Peeters, System identification and damage detection in civil engineering, Ph.D. thesis, Department of Civil Engineering, Katholieke Universiteit Leuven, Leuven, Belgium, 2000.

[13] C. H. J. Fox, "The location of defects in structures: a comparison of the use of natural frequency and mode shape data," in Proceedings of the 10th International Modal Analysis Conference, pp. 522-528, Society of Experimental Mechanics, Bethel, Conn, USA, 1992.

[14] O. S. Salawu and C. Williams, "Damage location using vibration mode shapes," in Proceedings of the 12th International Modal Analysis Conference, pp. 933-939, Society of Experimental Mechanics, Bethel, Conn, USA, 1994.

[15] A. K. Pandey, M. Biswas, and M. M. Samman, "Damage detection from changes in curvature mode shapes," Journal of Sound and Vibration, vol. 145, no. 2, pp. 321-332, 1991.

[16] Z. Zhang and A. E. Aktan, "The damage indices for constructed facilities," in Proceedings of the 13th International Modal Analysis Conference, pp. 1520-1529, Society of Experimental Mechanics, Bethel, Conn, USA, 1995.

[17] A. K. Pandey and M. Biswas, "Damage detection in structures using changes in flexibility," Journal of Sound and Vibration, vol. 169, no. 1, pp. 3-17, 1994.

[18] D. C. Zimmerman and M. Kaouk, "Structural damage detection using a minimum rank update theory," Journal of Vibration and Acoustics, vol. 116, no. 2, pp. 222-231, 1994.

[19] J.-T. Kim and N. Stubbs, "Model-uncertainty impact and damage-detection accuracy in plate girder," Journal of Structural Engineering, vol. 121, no. 10, pp. 1409-1417, 1995.

[20] J.-T. Kim and N. Stubbs, "Nondestructive crack detection algorithm for full-scale bridges," Journal of Structural Engineering, vol. 129, no. 10, pp. 1358-1366, 2003.

[21] P. Hajela and F. J. Soeiro, "Recent developments in damage detection based on system identification methods," Structural Optimization, vol. 2, no. 1, pp. 1-10, 1990.

[22] J. R. Casas and A. C. Aparicio, "Structural damage identification from dynamic-test data," Journal of Structural Engineering, vol. 120, no. 8, pp. 2437-2449, 1994.

[23] C. R. Farrar and T. A. Duffey, "Vibration-based damage detection in rotating machinery," Key Engineering Materials, vol. 167, pp. 224-235, 1999.

[24] D. L. Hunt, S. P. Weiss, W. M. West, T. A. Dunlap, and S. R. Freesmeyer, "Development and implementation of a shuttle modal inspection system," Sound and Vibration, vol. 24, no. 8, pp. 34-42, 1990.

[25] T. Toksoy and A. E. Aktan, "Bridge-condition assessment by modal flexibility," Experimental Mechanics, vol. 34, no. 3, pp. 271-278, 1994.
[26] C. R. Farrar, W. E. Baker, T. M. Bell, et al., "Dynamic characterization and damage detection in the I-40 bridge over the Rio Grande," Tech. Rep. LA 12767-MS, Los Alamos National Laboratory, Los Alamos, NM, USA, 1994.

[27] D. V. Jauregui and C. R. Farrar, "Comparison of damage identification algorithms on experimental modal data from a bridge," in Proceedings of the 14th International Modal Analysis Conference, pp. 1423-1429, Society of Experimental Mechanics, Bethel, Conn, USA, 1996.

[28] Z. Zhang and A. E. Aktan, "Application of modal flexibility and its derivatives in structural identification," Research in Nondestructive Evaluation, vol. 10, no. 1, pp. 43-61, 1998.

[29] F. N. Catbas and A. E. Aktan, "Condition and damage assessment: issues and some promising indices," Journal of Structural Engineering, vol. 128, no. 8, pp. 1026-1036, 2002.

[30] A. B. Siddique, B. F. Sparling, and L. D. Wegner, "Assessment of vibration-based damage detection for an integral abutment bridge," Canadian Journal of Civil Engineering, vol. 34, no. 3, pp. 438-452, 2007.

[31] B. Peeters, J. Maeck, and G. De Roeck, "Vibration-based damage detection in civil engineering: excitation sources and temperature effects," Smart Materials and Structures, vol. 10, no. 3, pp. 518-527, 2001.

[32] LabView, LabView ${ }^{T M}$ —version 6i, National Instruments Corporation, Austin, Tex, USA, 2000.

[33] R. Ramirez, The FFT: Fundamentals and Concepts, PrenticeHall, Englewood Cliffs, NJ, USA, 1985.

[34] M. E. Mortenson, Mathematics for Computer Graphics Applications, Industrial Press, New York, NY, USA, 1999.

[35] ANSYS, ANSYS User's Manual-version 7.1, ANSYS Inc., Canonsburg, Pa, USA, 2003.

[36] D. J. Ewins, Modal Testing: Theory, Practice and Applications, Research Studies Press, Hertfordshire, UK, 2nd edition, 2000.

[37] Y. K. Ho and D. J. Ewins, "On the structural damage identification with mode shapes," in Proceedings of the European COST F3 Conference on System Identification and Structural Health Monitoring, pp. 677-686, Madrid, Spain, 2000.

[38] N. Stubbs, Y. I. Kim, and C. R. Farrar, "Field verification of a nondestructive damage localization and severity estimation algorithm," in Proceedings of the 13th International Modal Analysis Conference, pp. 210-218, Society of Experimental Mechanics, Bethel, Conn, USA, 1995.

[39] C. R. Farrar and D. A. Jauregui, "Comparative study of damage identification algorithms applied to a bridge: I. Experiment," Smart Materials and Structures, vol. 7, no. 5, pp. 704-719, 1998.

[40] S. Choi, S. Park, and N. Stubbs, "Nondestructive damage detection in structures using changes in compliance," International Journal of Solids and Structures, vol. 42, no. 15, pp. 4494-4513, 2005. 

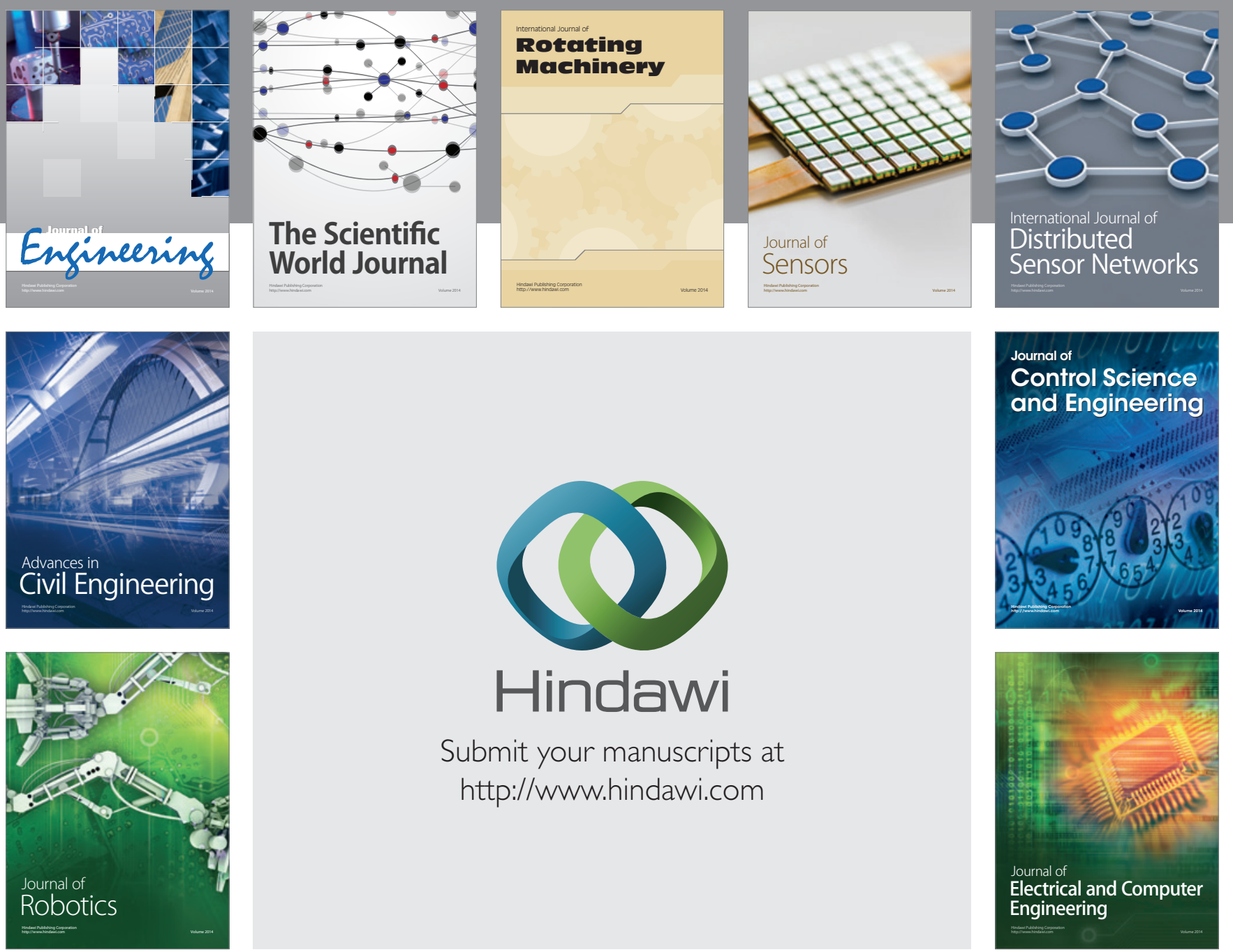

Submit your manuscripts at

http://www.hindawi.com
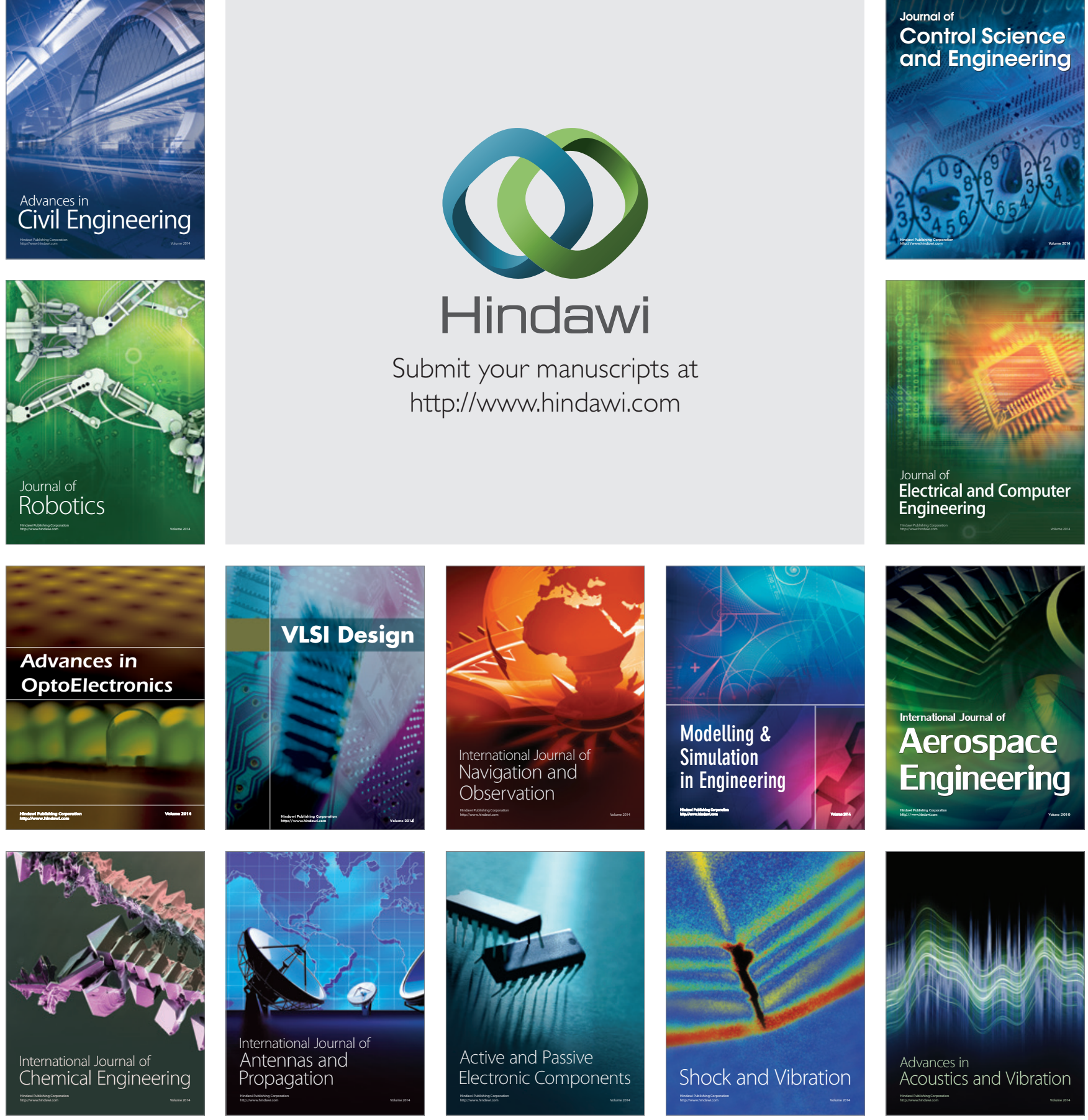